\title{
Recursive dynamic mode decomposition of transient and post-transient wake flows
}

\author{
Bernd R. Noack ${ }^{1,2, \dagger}$, Witold Stankiewicz ${ }^{3}$, Marek Morzyński ${ }^{3}$ \\ and Peter J. Schmid ${ }^{4}$ \\ ${ }^{1}$ Laboratoire d'Informatique pour la Mécanique et les Sciences de l'Ingénieur, LIMSI-CNRS, \\ Rue John von Neumann, Campus Universitaire d'Orsay, Bât 508, F-91403 Orsay, France \\ ${ }^{2}$ Institut für Strömungsmechanik, Technische Universität Berlin, Hermann-Blenk-Straße 37, \\ D-38108 Braunschweig, Germany \\ ${ }^{3}$ Chair of Virtual Engineering, Poznań University of Technology, ul. Jana Pawła II 24, \\ 60-965 Poznań, Poland \\ ${ }^{4}$ Department of Mathematics, Imperial College London, South Kensington Campus, \\ London SW7 2AZ, UK
}

(Received 21 November 2015; revised 31 July 2016; accepted 19 October 2016; first published online 21 November 2016)

A novel data-driven modal decomposition of fluid flow is proposed, comprising key features of proper orthogonal decomposition (POD) and dynamic mode decomposition (DMD). The first mode is the normalized real or imaginary part of the DMD mode that minimizes the time-averaged residual. The $N$ th mode is defined recursively in an analogous manner based on the residual of an expansion using the first $N-1$ modes. The resulting recursive DMD (RDMD) modes are orthogonal by construction, retain pure frequency content and aim at low residual. Recursive DMD is applied to transient cylinder wake data and is benchmarked against POD and optimized DMD (Chen et al., J. Nonlinear Sci., vol. 22, 2012, pp. 887-915) for the same snapshot sequence. Unlike POD modes, RDMD structures are shown to have purer frequency content while retaining a residual of comparable order to POD. In contrast to DMD, with exponentially growing or decaying oscillatory amplitudes, RDMD clearly identifies initial, maximum and final fluctuation levels. Intriguingly, RDMD outperforms both POD and DMD in the limit-cycle resolution from the same snapshots. Robustness of these observations is demonstrated for other parameters of the cylinder wake and for a more complex wake behind three rotating cylinders. Recursive DMD is proposed as an attractive alternative to POD and DMD for empirical Galerkin models, in particular for nonlinear transient dynamics.

Key words: low-dimensional models, wakes

\section{Introduction}

This study proposes a novel flow field expansion tailored to the construction of lowdimensional empirical Galerkin models. Such reduced-order models (1) help in data compression, (2) allow quick visualizations and kinematic mixing studies (Rom-Kedar,

$\dagger$ Email address for correspondence: bernd.noack@limsi.fr 
Leonard \& Wiggins 1990), (3) provide a testbed for physical understanding (Lorenz 1963), (4) serve as computationally inexpensive surrogate models for optimization (Han, Stefan \& Zimmermann 2013) or (5) may be used as a plant for control design (Gerhard et al. 2003; Bergmann \& Cordier 2008).

In 1858, Helmholtz laid the foundation for the first low-dimensional dynamical models in fluid mechanics with his famous theorems on vortices (see, e.g., Lugt 1995). Subsequently, a rich set of vortex models has been developed for vortex pairs, for the recirculation zone (Föppl 1913; Suh 1993), for the vortex street (von Kármán \& Rubach 1912) and for numerous combustion related problems (Coats 1997), to name just a few. For non-periodic open flows, low-dimensional vortex models come at the expense of a hybrid state-space structure: new degrees of freedom (vortices) are created at the body, merged or removed. Most forms of applications, like dynamical systems analyses or control design, are not suitable for hybrid models but assume a continuous evolution in a fixed finite-dimensional state space. Hence, most currently developed reduced-order models are formulated by the Galerkin method and based on modal expansions (Fletcher 1984).

In the last 100 years, Galerkin (1915)'s method of solving partial differential equations has enjoyed ample generalizations and applications, from high-dimensional grid-based computational methods to low-dimensional models. This study focuses on low-dimensional flow representations by an expansion in global modal structures. In principle, any space of square-integrable velocity fields has a complete set of orthonormal modes: any flow field can be arbitrarily closely approximated by a finite-dimensional expansion. In practice, however, the construction of such mathematical bases is restricted to simple geometries and the use of Fourier expansions or Chebyshev polynomials (Orszag 1971). Stability modes based on a linearization of the Navier-Stokes equations tend to be more efficient in terms of resolution for a given number $N$ of modes. However, these physical modes generally lack a proof of completeness, and are afflicted by a reduced dynamic bandwidth and an $O\left(N^{3}\right)$ operation count for the quadratic terms, as opposed to $O(N \log N)$ operations for Fourier or Chebyshev modes. The most efficient representations of a Navier-Stokes solution are obtained from empirical expansions based on flow snapshots. These data-driven Galerkin expansions are confined to a subspace spanned by the snapshots, i.e. have a narrow dynamic bandwidth defined by the training set.

This study focuses on data-driven expansions. A Galerkin expansion with guaranteed minimal residual over the training snapshots was first pioneered by Lorenz (1956) as principal axis modes and later popularized in fluid mechanics as proper orthogonal decomposition (POD) by Lumley (1967). Proper orthogonal decomposition guarantees an optimal data reconstruction in a well-defined sense (see, e.g., Holmes, Lumley \& Berkooz 1998). Apart from data compression applications, Lumley devised POD as a mathematical tool for distilling coherent structures from data. Yet, only in rare cases have POD modes been found to resemble physically meaningful structures, like stability modes or base-flow deformations (Oberleithner et al. 2011). In particular, they tend to mix spatial and temporal frequencies in most modes, which complicates a physical interpretation. As a remedy for this shortcoming, the dynamic mode decomposition (DMD) - also referred to as Koopman analysis - was pioneered by Rowley et al. (2009) and Schmid (2010). Dynamic mode decomposition is able to distil stability eigenmodes from transient snapshot data and produce temporal Fourier modes for post-transient data. The downside of these DMD features - compared with POD - is non-orthogonality of the extracted modes, suboptimal convergence, the need for time-resolved snapshots and numerical challenges when filtering is omitted. Mezić (2013) provides an excellent review of recent DMD developments. 
In the past, numerous Galerkin models based on POD and DMD have been constructed. In addition, numerous generalizations have been proposed to address, among other topics, multi-operating conditions (Jørgensen, Sørensen \& Brøns 2003), changes during transients (Siegel et al. 2008), optimal correlations to observables (Hoarau et al. 2006; Schlegel et al. 2012) and control design (Brunton \& Noack 2015).

This study proposes a novel data-driven expansion preserving key features of POD, such as orthonormality of the computed modes and a low residual, and of DMD, such as distilling the dominant frequencies and their associated structures contained in the data. The paper is organized as follows. Section 2 describes the cylinder wake configuration and gives details on the direct numerical simulation employed as well as the snapshots extracted. Section 3 outlines the computation of the proposed expansion, called 'recursive DMD (RDMD)' in what follows. Recursive DMD is then applied to a transient cylinder wake, demonstrating its advantages $(\S 4)$ over previous decompositions. In $\S 5$, a similar investigation is performed for the non-periodic wake behind three rotating cylinders. Our results are summarized in $\S 6$.

\section{Configuration and direct numerical simulation}

As a test case, a two-dimensional incompressible viscous flow past a circular cylinder has been chosen. The flow is described in a Cartesian coordinate system where the $x$-axis is aligned with the streamwise direction and the $y$-axis is transverse and orthogonal to the cylinder axis. The origin of the coordinate system coincides with the cylinder axis. The location vector is denoted by $\boldsymbol{x}=(x, y)$. Analogously, the velocity is represented by $\boldsymbol{u}=(u, v)$, where $u$ and $v$ are the $x$ - and $y$-components of the velocity. The time is represented by $t$. The Newtonian fluid is characterized by the density $\rho$ and dynamic viscosity $\mu$. In the following, all variables are assumed to be non-dimensionalized by the cylinder diameter $D$, the oncoming velocity $U$ and the fluid density $\rho$. The resulting Reynolds number $R e=U D \rho / \mu$ is set to 100 , i.e. well above the onset of vortex shedding (Zebib 1987; Schumm, Berger \& Monkewitz 1994), but well below the onset of three-dimensional instabilities (Zhang et al. 1995; Barkley \& Henderson 1996; Williamson 1996).

A direct numerical simulation of the Navier-Stokes equations has been performed using an in-house solver based on a second-order finite-element discretization with Taylor-Hood elements (Taylor \& Hood 1973) in primitive variables. The time stepping is performed using a third-order-accurate scheme and a time step equal to 0.1. Following Noack et al. (2003), the computational domain extends from $x=-5$ to $x=25$ and $y=-5$ to $y=5$ and is discretized with 56272 finite elements. The initial condition for the transient from $t=0$ to $t=150$ is a small perturbation of the steady Navier-Stokes solution $\boldsymbol{u}_{s}$ and reads $\boldsymbol{u}(\boldsymbol{x}, 0)=\boldsymbol{u}_{s}(\boldsymbol{x})+0.01 \boldsymbol{f}_{1}(\boldsymbol{x})$, where $\boldsymbol{f}_{1}$ is the real part of the unstable complex eigenvector normalized to unit norm.

The simulation provides $M=450$ equidistantly sampled velocity snapshots $\boldsymbol{u}^{m}(\boldsymbol{x})=$ $\boldsymbol{u}\left(\boldsymbol{x}, t^{m}\right), m=1, \ldots, M$, covering the entire unforced transient phase, from the steady solution to the fully developed von Kármán vortex street. The snapshot times are $t^{m}=30+m \Delta t$ based on a sampling interval of $\Delta t=0.2$ and cover the time interval $t \in[30,120]$. The initial and final base flows are depicted in figure 1 together with the energy norm of the fluctuations $\boldsymbol{v}=\boldsymbol{u}-\boldsymbol{u}_{s}$ around the steady solution $\boldsymbol{u}_{s}$. The transition from the unstable fixed point to the stable limit-cycle oscillation is characterized by the growing amplitude of the fluctuations accompanied by an increase in the Strouhal number (Zebib 1987; Schumm et al. 1994). 
(a)

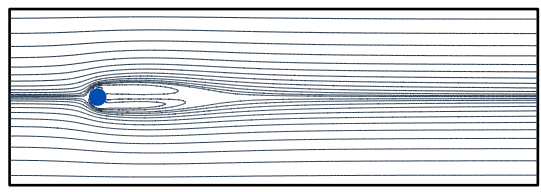

(b)

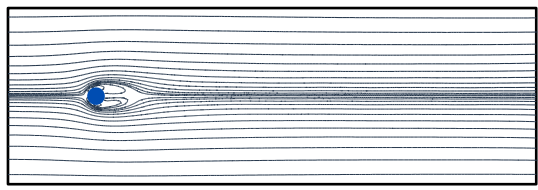

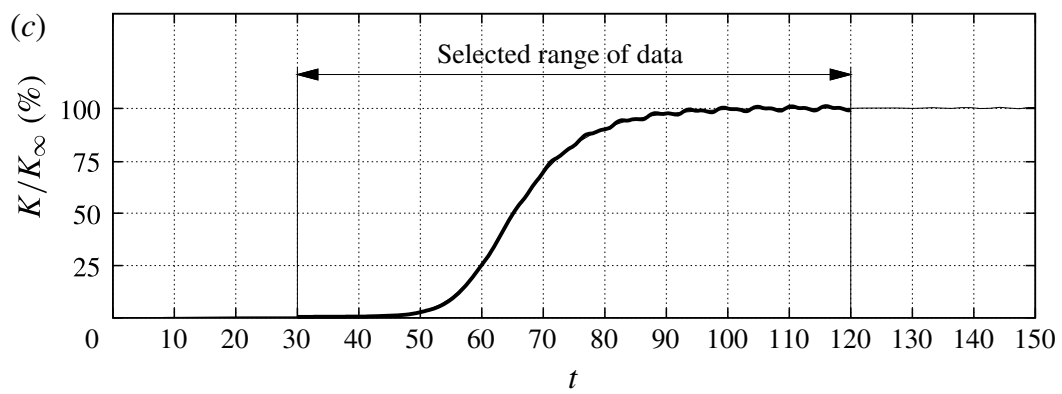

FIgURE 1. (Colour online) Incompressible two-dimensional flow around a circular cylinder at $R e=100$. The steady $(a)$ and time-averaged $(b)$ solutions are illustrated with streamlines. The total fluctuation level $K$ normalized with its asymptotic value $K_{\infty}$ is shown in $(c)$.

Within the first 30 convective time units, the flow is governed by linear dynamics spanned by the steady solution and the unstable eigenmode. In the intermediate phase, approximately $t \in[60,90]$, the vortex shedding undergoes significant changes and moves farther upstream towards the cylinder. In the final 30 convective time units, the flow has converged to a limit-cycle dynamics exhibiting a fully developed von Kármán vortex street. These three characteristic stages of the transient evolution are depicted in figure 2. For the construction of the Galerkin expansions, we ignore the phase $t \in[0,30]$ as there is hardly any noticeable fluctuation and the corresponding snapshots effectively duplicate the steady Navier-Stokes solution. We also ignore the converged limit-cycle data at $t>120$ as this would also give emphasis to redundant information.

All modal decompositions are based on these fluctuations around the steady solution. The mean flow is discarded as a base flow because it is only well defined for this particular initial condition and the chosen time interval. Our sampling Strouhal frequency of 10 is approximately 30 times larger than the shedding frequency - a value that can be considered adequate for a Fourier transformation while avoiding excessive redundancy for the statistical POD.

\section{Modal decomposition}

In this section, a new snapshot-based modal decomposition is proposed. This decomposition comprises properties of the POD (Lumley 1967; Sirovich 1987) and the DMD (Rowley et al. 2009; Schmid 2010). First ( $\$ 3.1$ and 3.2), the snapshot-based POD and DMD are briefly recapitulated. In $\S 3.3$, the RDMD is proposed as an appealing compromise inheriting the orthonormality and low truncation error of POD and the oscillatory representation of the flow behaviour of DMD.

\subsection{Proper orthogonal decomposition}

We analyse a time-dependent velocity field $\boldsymbol{u}(\boldsymbol{x}, t)$ in a steady domain $\boldsymbol{x} \in \Omega$ and sample $M$ flow snapshots with constant sampling frequency corresponding to a time 
(a)

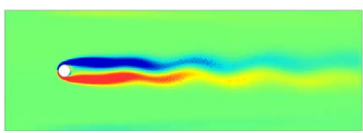

(b)

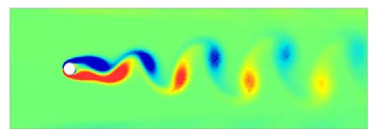

(c)



FIGURE 2. (Colour online) Transient evolution of the cylinder wake illustrated with snapshots of the vorticity field at an initial $(a, t=50)$, an intermediate $(b, t=70)$ and a final state $(c, t=90)$.

step $\Delta t$. The velocity field at instant $t^{m}=m \Delta t$, with $m=1, \ldots, M$, is denoted by $\boldsymbol{u}^{m}:=\boldsymbol{u}\left(\boldsymbol{x}, t^{m}\right)$.

Proper orthogonal decomposition requires the definition of an inner product and a time average. We assume the standard inner product of two square-integrable velocity fields $\boldsymbol{u}^{1}, \boldsymbol{u}^{2} \in \mathcal{L}^{2}(\Omega)$, given as

$$
\left(\boldsymbol{u}^{1}, \boldsymbol{u}^{2}\right)_{\Omega}:=\int_{\Omega} \mathrm{d} \boldsymbol{x} \boldsymbol{u}^{1}(\boldsymbol{x}) \cdot \boldsymbol{u}^{2}(\boldsymbol{x}) .
$$

The corresponding norm reads $\|\boldsymbol{u}\|_{\Omega}:=\sqrt{(\boldsymbol{u}, \boldsymbol{u})_{\Omega}}$. The snapshot-based time average of a function $F$ is defined in a canonical manner,

$$
\langle F(\boldsymbol{u})\rangle_{M}:=\frac{1}{M} \sum_{m=1}^{M} F\left(\boldsymbol{u}^{m}\right) .
$$

In the following, the snapshot-based POD (Sirovich 1987) is applied to the fluctuations

$$
\boldsymbol{v}^{m}(\boldsymbol{x}):=\boldsymbol{u}\left(\boldsymbol{x}, t^{m}\right)-\boldsymbol{u}_{s}(\boldsymbol{x})
$$

around the steady solution $\boldsymbol{u}_{s}$ for the reasons mentioned in $\S 2$. First, the correlation matrix $\boldsymbol{C}=\left(C^{m n}\right) \in \mathbb{R}^{M \times M}$ of the fluctuations is determined,

$$
C^{m n}:=\frac{1}{M}\left(\boldsymbol{v}^{m}, \boldsymbol{v}^{n}\right)_{\Omega}
$$

Second, a spectral analysis of this matrix is performed. It should be noted that $\boldsymbol{C}$ is a symmetric positive semi-definite Gramian matrix. Hence, its eigenvalues $\lambda_{i}$ are real and non-negative and can be assumed to be sorted according to $\lambda_{1} \geqslant \lambda_{2} \geqslant \ldots \geqslant \lambda_{M} \geqslant 0$. The corresponding eigenvectors $\boldsymbol{e}_{i}=\left[e_{i}^{1}, \ldots, e_{i}^{M}\right]^{\mathrm{T}}$ satisfy

$$
\boldsymbol{C} \boldsymbol{e}_{i}=\lambda_{i} \boldsymbol{e}_{i}, \quad i=1, \ldots, M,
$$

and can - without loss of generality - be assumed to be orthonormalized, satisfying $\boldsymbol{e}_{i} \cdot \boldsymbol{e}_{j}=\delta_{i j}$, with $\delta_{i j}$ as the Kronecker symbol. Third, each POD mode is expressed as a linear combination of the snapshot fluctuations,

$$
\boldsymbol{u}_{i}:=\frac{1}{\sqrt{M \lambda_{i}}} \sum_{m=1}^{M} e_{i}^{m} \boldsymbol{v}^{m}, \quad i=1, \ldots, N .
$$

It follows that the POD modes are orthonormal, with $\left(\boldsymbol{u}_{i}, \boldsymbol{u}_{j}\right)_{\Omega}=\delta_{i j}, \quad \forall i, j \in$ $\{1, \ldots, M\}$. Finally, the mode amplitudes read

$$
a_{i}^{m}:=\sqrt{\lambda_{i} M} e_{m}^{i}, \quad i=1, \ldots, N .
$$


These amplitudes are uncorrelated (orthogonal in time), or in mathematical terms

$$
\left\langle a_{i} a_{j}\right\rangle_{M}=\lambda_{i} \delta_{i j}, \quad i, j \in\{1, \ldots, N\}
$$

It should be noted that the first moments $\left\langle a_{i}\right\rangle_{M}$ do not need to vanish as the fluctuations are based on the steady solution and not on the mean flow of this transient. The POD defines a second-order statistics providing the two-point autocorrelation function

$$
\overline{\boldsymbol{v}(\boldsymbol{x}, t) \boldsymbol{v}(\boldsymbol{y}, t)}=\sum_{i=1}^{N} \lambda_{i} \boldsymbol{u}_{i}(\boldsymbol{x}) \boldsymbol{u}_{i}(\boldsymbol{y}) .
$$

Hence, a minimum requirement imposed on the snapshot ensemble is the accuracy of the extracted mean flow and the second moments of the flow. This accuracy of the statistics for a given number of snapshots is increased by processing uncorrelated snapshots, as required in the original paper on the snapshot POD method (Sirovich 1987).

The resulting expansion exactly reproduces the snapshots for $N=M$ modes; we have

$$
\boldsymbol{u}\left(\boldsymbol{x}, t^{m}\right)=\boldsymbol{u}_{s}(\boldsymbol{x})+\sum_{i=1}^{N} a_{i}\left(t^{m}\right) \boldsymbol{u}_{i}(\boldsymbol{x}) .
$$

For $N<M$, the truncated expansion (3.10) has a non-vanishing residual $\boldsymbol{r}^{m}(\boldsymbol{x}):=$ $\boldsymbol{r}\left(\boldsymbol{x}, t^{m}\right)$. The corresponding time-averaged truncation error

$$
\chi^{2}:=\left\langle\left\|\boldsymbol{r}^{m}\right\|_{\Omega}^{2}\right\rangle_{M}
$$

can be shown to be minimal; in other words, no other Galerkin expansion with the same number of modes will have a smaller error (Holmes et al. 1998). This optimality property makes POD an attractive data compression technique.

For later reference, the instantaneous truncation error $\chi^{2}(t):=\left\|\boldsymbol{r}^{m}(\cdot, t)\right\|_{\Omega}^{2}$ is introduced. The size of this error may be compared with the corresponding fluctuation level on the limit cycle $2 K_{\infty}=\overline{\|v\|^{2}}$, where $K_{\infty}$ denotes the turbulent kinetic energy (TKE) and the overbar represents averaging over the post-transient phase. We also introduce $K$ as the corresponding instantaneous quantity.

As a motivation for the proposed new decomposition, we recall that POD can also be defined in a recursive manner, following Courant \& Hilbert (1989) on the spectral analysis of positive definite symmetric matrices. Taking $\boldsymbol{u}_{1}$ as the first expansion mode, the resulting one-mode expansion reads

$$
\boldsymbol{v}^{m}=a_{1}^{m} \boldsymbol{u}_{1}+\boldsymbol{r}_{1}^{m}, \quad m=1, \ldots, M .
$$

The mode amplitude $a_{1}^{m}:=\left(\boldsymbol{v}^{m}, \boldsymbol{u}_{1}\right)_{\Omega}$ minimizes the residual $\left\|\boldsymbol{r}_{1}^{m}\right\|_{\Omega}$ for a given $\boldsymbol{u}_{1}$. The first POD mode can be shown to minimize the averaged energy of the residual $\left\langle\left\|\boldsymbol{r}_{1}^{m}\right\|_{\Omega}^{2}\right\rangle_{M}$. Furthermore, the residual $\boldsymbol{r}_{1}^{m}, m=1, \ldots, M$ is orthogonal to $\boldsymbol{u}_{1}$ by construction. The second step then searches for a mode $\boldsymbol{u}_{2}$ that best resolves the residual $\boldsymbol{r}_{1}$,

$$
\boldsymbol{r}_{1}^{m}=a_{2}^{m} \boldsymbol{u}_{2}+\boldsymbol{r}_{2}^{m}, \quad m=1, \ldots, M,
$$

i.e. that minimizes $\left\langle\left\|\boldsymbol{r}_{2}^{m}\right\|_{\Omega}^{2}\right\rangle_{M}$. The other remaining modes are computed in a similar manner. 


\subsection{Dynamic mode decomposition}

Dynamic mode decomposition (Rowley et al. 2009; Schmid 2010) is another data-driven modal expansion which can approximate stability eigenmodes from transient data or Fourier modes from post-transient data. The time step $\Delta t$ needs to be sufficiently small for a meaningful Fourier analysis, but sufficiently large so that the changes in the flow state exceed the noise level.

We consider the fluctuation snapshots of $\S 3.1, \boldsymbol{v}^{m}, m=1, \ldots, M-1$, as linearly independent modes of an expansion and write

$$
\boldsymbol{v}=\sum_{i=1}^{M-1} b_{i} \boldsymbol{v}^{i}
$$

Evidently, the modes are generally not orthogonal. With this basis, the mode amplitude vector of the $m$ th snapshot becomes a unit vector,

$$
\boldsymbol{b}^{m}=\left[b_{1}^{m}, \ldots, b_{M-1}^{m}\right]^{\mathrm{T}}, \quad \text { where } b_{i}^{m}=\delta_{i m} .
$$

Dynamic mode decomposition assumes a linear relationship between the $(m+1)$ th and $m$ th snapshot,

$$
\boldsymbol{b}^{m+1}=\boldsymbol{A} \boldsymbol{b}^{m}
$$

where $\boldsymbol{A} \in \mathbb{R}^{(M-1) \times(M-1)}$ is a square matrix which is generally identified from the data.

For $m<M-1$, (3.16) acts as a shift map: the $m$ th unit vector is mapped on the $(m+1)$ th unit vector, leading to unity in the first subdiagonal of $\boldsymbol{A}$ and zeros in the remaining column. The $M$ th snapshot has no successor in the time series and is expanded in terms of the previous snapshots,

$$
\boldsymbol{v}^{M}=\sum_{i=1}^{M-1} c_{i} \boldsymbol{v}^{i}+\boldsymbol{r}
$$

The coefficients $c_{i}$ are chosen to minimize the residual norm $\|\boldsymbol{r}\|_{\Omega}$.

From (3.16) and (3.17), the matrix is easily seen to be of companion type,

$$
\boldsymbol{A}=\left[\begin{array}{cccccc}
0 & 0 & \ldots & 0 & 0 & c_{1} \\
1 & 0 & \ldots & 0 & 0 & c_{2} \\
\vdots & \vdots & \ddots & \vdots & \vdots & \\
0 & 0 & \ldots & 1 & 0 & c_{M-2} \\
0 & 0 & \ldots & 0 & 1 & c_{M-1}
\end{array}\right]
$$

In what follows, we depart from the classical DMD literature and propose a simpler derivation of the DMD modes. Let $P(s)=c_{1}+c_{2} s+\cdots+c_{M-1} s^{M-2}+s^{M-1}$ be a polynomial in $s$ and let $P(s)=\left(s-\lambda_{1}\right)\left(s-\lambda_{2}\right) \cdots\left(s-\lambda_{M-1}\right)$ be its factorization with distinct eigenvalues $\lambda_{i}$. We introduce

$$
\boldsymbol{V}=\left[\begin{array}{cccc}
1 & \lambda_{1} & \ldots & \lambda_{1}^{M-2} \\
1 & \lambda_{2} & \ldots & \lambda_{2}^{M-2} \\
\vdots & \vdots & \vdots & \vdots \\
1 & \lambda_{M-1} & \ldots & \lambda_{M-1}^{M-2}
\end{array}\right]
$$


as the corresponding Vandermonde matrix. It can be easily verified that the Vandermonde matrix $\boldsymbol{V}$ diagonalizes the companion matrix $\boldsymbol{A}$. We obtain

$$
\boldsymbol{V} \boldsymbol{A} \boldsymbol{V}^{-1}=\operatorname{diag}\left(\lambda_{1}, \ldots, \lambda_{M-1}\right),
$$

where the right-hand side is a diagonal matrix with the eigenvalues as its elements. We introduce new variables $\boldsymbol{a}$ defined by

$$
\boldsymbol{a}=\boldsymbol{V} \boldsymbol{b} .
$$

With these definitions, the evolution equation (3.16) can be cast into eigenform according to

$$
\boldsymbol{a}^{m+1}=\boldsymbol{V} \boldsymbol{C} \boldsymbol{V}^{-1} \boldsymbol{a}^{m}=\boldsymbol{D} \boldsymbol{a}^{m} .
$$

Here, $\boldsymbol{D}$ denotes the diagonal matrix

$$
\boldsymbol{D}=\operatorname{diag}\left(\lambda_{1}, \ldots, \lambda_{M-1}\right)=\left[\begin{array}{cccc}
\lambda_{1} & 0 & \ldots & 0 \\
0 & \lambda_{2} & \ldots & 0 \\
\vdots & \vdots & \vdots & \vdots \\
0 & 0 & \ldots & \lambda_{M-1}
\end{array}\right]
$$

where the $i$ th eigenvalue is $\lambda_{i}$, with corresponding eigenvector $\boldsymbol{a}_{i}=\left[\delta_{1 i}, \ldots, \delta_{(M-1) i}\right]^{\mathrm{T}}$.

Equations (3.15), (3.16) and (3.21) imply that the snapshots can be expressed as

$$
\boldsymbol{v}^{m}=\sum_{i=1}^{M-1} \lambda_{i}^{m-1} \boldsymbol{\Phi}_{i}
$$

where the $\lambda_{i}$ are referred to as DMD eigenvalues and the $\boldsymbol{\Phi}_{i}$ as (complex) DMD modes. It should be noted that the derivation of (3.24) rests on the diagonalization of the companion matrix (3.20) and does not require the Koopman operator.

The choice of $N$ DMD modes for the Galerkin expansion (3.10) minimizes the truncation error (3.11) following Chen, Tu \& Rowley (2012) and consistent with the optimal property of POD.

\subsection{Recursive DMD}

Recursive DMD serves a multi-objective task: extracting oscillatory modes from the snapshot sequence (like DMD) while ensuring orthogonality of the modes and a low truncation error (like POD).

The initialization step prepares the residual to be processed. We take

$$
\boldsymbol{r}_{0}^{m}:=\boldsymbol{v}^{m}, \quad m=1, \ldots, M .
$$

During the $i$ th step $(0<i \leqslant N)$, the $i$ th mode is determined from a DMD

$$
\boldsymbol{r}_{i-1}^{m}=\sum_{j=1}^{M-1} \lambda_{j}^{m-1} \boldsymbol{\Phi}_{j}^{i}
$$

where $\boldsymbol{\Phi}_{j}^{i}$ represents the $j$ th DMD mode of the $i$ th step. The candidate modes to be considered are

$$
\boldsymbol{u}_{k}^{\star}=\frac{\mathfrak{R}\left\{\boldsymbol{\Phi}_{k}^{i}\right\}}{\left\|\mathfrak{R}\left\{\boldsymbol{\Phi}_{k}^{i}\right\}\right\|_{\Omega}}, \quad k=1, \ldots, M-1,
$$


and each mode reduces the residual $\boldsymbol{r}_{i, k}^{m}$ according to

$$
\begin{aligned}
\boldsymbol{r}_{i-1}^{m} & =a_{k}^{\star m} \boldsymbol{u}_{k}^{\star}+\boldsymbol{r}_{i, k}^{m}, \\
a_{k}^{\star m} & =\left(\boldsymbol{r}_{i-1}^{m} \cdot \boldsymbol{u}_{k}^{\star}\right)_{\Omega} .
\end{aligned}
$$

It should be noted that the residual $\boldsymbol{r}_{i, k}^{m}$ depends on the index of iteration $i$, the snapshot index $m$ and the trial mode index $k$. The truncation error of the $i$ th candidate mode $\boldsymbol{u}_{k}^{\star}$ for all snapshots is given by

$$
\chi_{i, k}^{2}:=\left\langle\left\|\boldsymbol{r}_{i, k}^{m}\right\|_{\Omega}^{2}\right\rangle_{M} .
$$

As mode $i$ we select the trial mode $k$ with the lowest averaged error, i.e.

$$
\boldsymbol{u}_{i}:=\boldsymbol{u}_{k}^{\star} \quad \text { so that } \quad \forall l \in\{1, \ldots, M-1\}: \chi_{i, k}^{2} \leqslant \chi_{i, l}^{2} .
$$

The resulting expansion reads after the $i$ th step

$$
\boldsymbol{v}^{m}:=\sum_{j=1}^{i} a_{j} \boldsymbol{u}_{j}+\boldsymbol{r}_{i}^{m} .
$$

The $(i+1)$ th mode is computed following the same steps. The iteration terminates when the desired number of modes is reached, $i=N$, or in the unlikely case that all residuals vanish, $\boldsymbol{r}_{i}^{m} \equiv 0, \quad m=1, \ldots, M-$ whichever criterion is satisfied first. We emphasize that the candidate modes are normalized (see (3.27)) and the $(i+1)$ th mode is constructed from the orthogonal residual of the Galerkin expansion with the first $i$ modes (see (3.28)). Hence, the modes form an orthonormal system.

\section{Modal decomposition of the transient cylinder wake}

The transient cylinder wake is analysed with POD (§4.1), DMD (§4.2) and the proposed new decomposition ( $\$ 4.3$ ) discussed in $\S 3$. In $\S 4.4$, all modal decompositions are subjected to a comparison with respect to the instantaneous residual, the averaged residual and the convergence with increasing number of modes. The transient wake snapshots are the same for all decompositions and have been described in $\S 2$.

\subsection{Proper orthogonal decomposition}

The snapshots of the cylinder wake simulations described in $\S 2$ are subjected to a snapshot POD outlined in $\$ 3.1$. The POD modes of the post-transient periodic cylinder wake mimic a Fourier decomposition. They arise in pairs representing the two phases at the first and higher harmonic frequencies (Deane et al. 1991; Noack et al. 2003). The energy level of each pair rapidly decreases with the order of the harmonics. The transient, however, exhibits a gradual change from the initial stability modes (with a fluctuation maximum far from the cylinder) to von Kármán vortex shedding (with fluctuations peaking near the cylinder). The other change is a base flow with a recirculation region that decreases in streamwise extent from approximately 7 diameters to approximately 1 diameter length. The resulting POD modes and associated mode amplitudes are depicted in figures 3 and 4 and appear to be different from the post-transient analogues. Proper orthogonal decomposition 
(a)

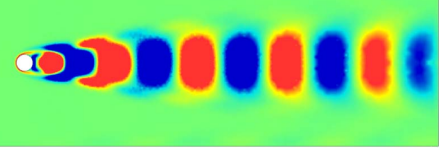

(c)

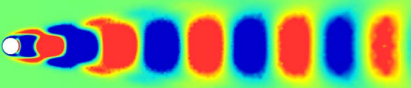

(e)

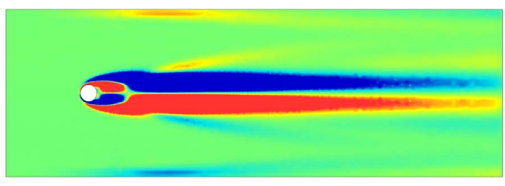

(

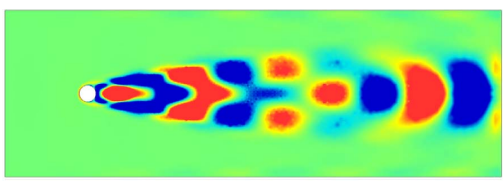

(i)



(b)

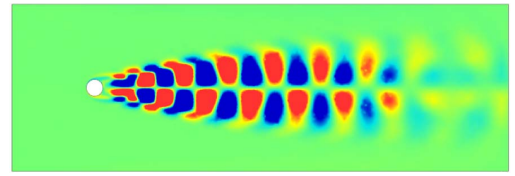

(d)

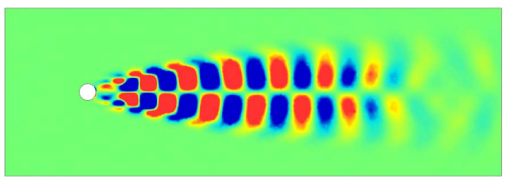

$(f)$

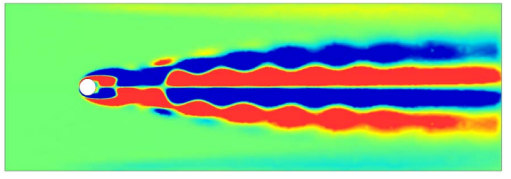

(h)

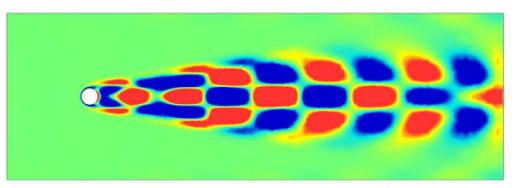

(j)

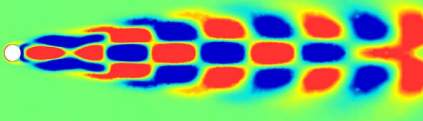

Figure 3. (Colour online) The first 10 POD modes of the transient phase of the flow, with $t \in[30,120]$ and $R e=100$. (a,c,e,g,i) Modes $1-5,(b, d, f, h, j)$ modes 6-10. The vorticity of the mode is visualized in colour (green, zero; red, above a positive threshold; blue, below a negative threshold).

modes 1 and 2 effectively represent von Kármán vortex shedding growing from zero to asymptotic values. These values are larger than the corresponding fluctuation levels of the other modes. Modes 6 and 7 describe the second harmonics, as can be seen from the visualization and the amplitude evolution. Modes 9 and 10 look similar to the stability eigenmodes at slightly lower frequencies, with peak activity around $t=55$. Modes 4 and 5 also represent vortex shedding, with peak activity around $t=65$, i.e. two shedding periods later. Mode 3 depicts the shift mode (Noack et al. 2003), i.e. it characterizes the base-flow change between steady and time-averaged periodic solution. Mode 8 represents another base-flow correction with different topology and is mainly active during the most rapid changes of the transient around $t=65$. It has a small second harmonic component. The base-flow modes 3 and 8 correspond to an expansion with the steady solution as basic modes. Taking the post-transient averaged velocity as basic mode is numerically found to give rise to similar base-flow modes but with different energy content and thus different indices.

All of the displayed POD modes show pronounced frequencies: six modes display oscillations near the shedding frequency, two resolve the second harmonics and two feature slowly varying base-flow changes. Unlike POD for periodic shedding, the third and higher harmonics are found as modes with indices after the first 10 
(a)

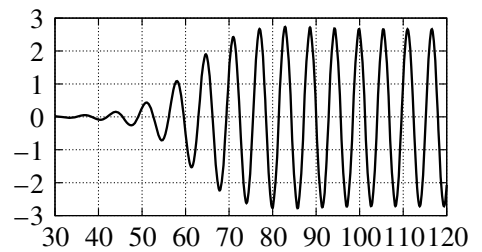

(c)

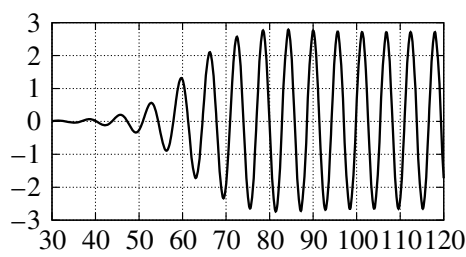

(e)



$(g)$

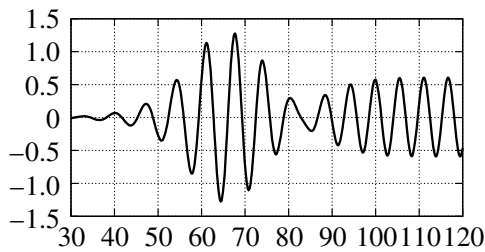

(i)

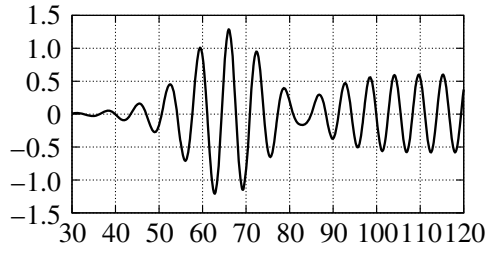

(b)



(d)

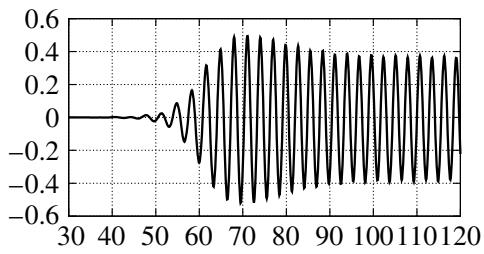

$(f)$

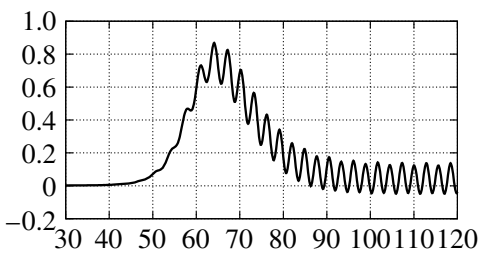

(h)

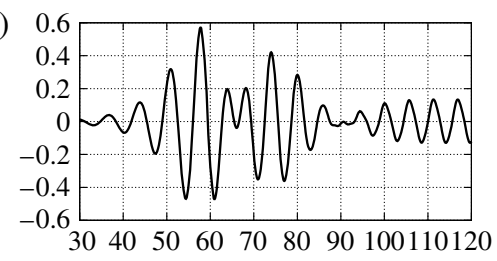

(j)

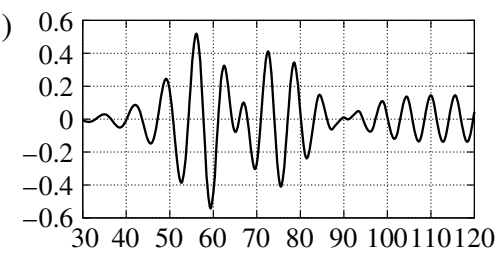

FIgURE 4. The mode amplitudes of the POD modes of figure 3.

ones displayed. This comparatively 'clean' frequency content may be attributed to the narrow-bandwidth transient dynamics, with dominant frequencies between the eigenfrequency of the steady solution and the shedding frequency of the post-transient wake. Moreover, the maximum of the fluctuation envelope moves upstream during the transient. For broadband dynamics, such as a mixing layer with multiple vortex pairings, POD modes with multiple frequency content are far more common (Noack et al. 2004).

\subsection{Dynamic mode decomposition}

The snapshots of the transient flow have been decomposed with DMD. The DMD procedure can identify eigenmodes for the transient data and the Fourier modes for the attractor. 
(a)

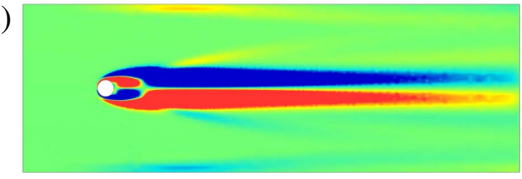

(c)

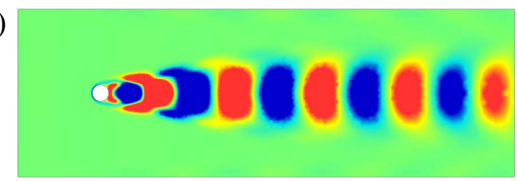

(e)

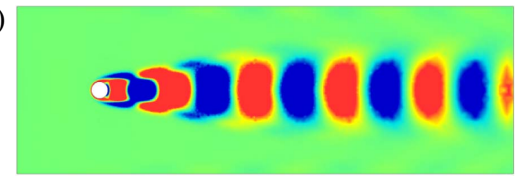

(g)



(i)

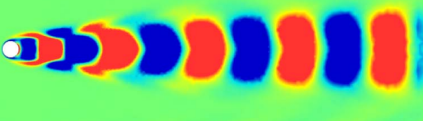

(b)

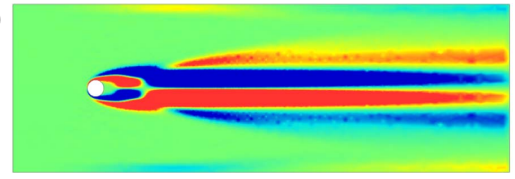

(d)

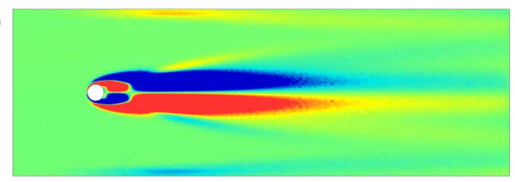

(f)

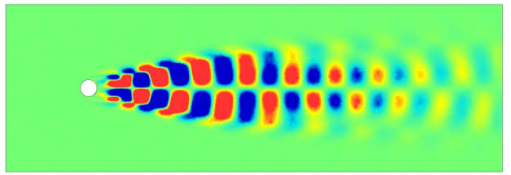

(h)

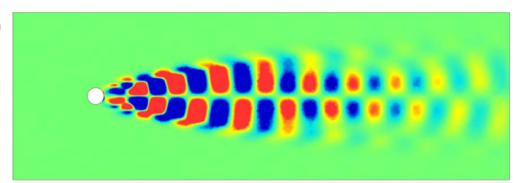

(j)

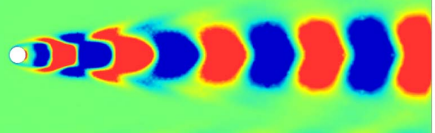

FIgURE 5. (Colour online) The same as figure 3 but for the first 10 DMD modes.

The result of the procedure is a set of complex Ritz vectors and complex eigenvalues characterizing the growth rate and the frequency of the respective mode. The first 10 eigenmodes are depicted in figure 5. The first DMD mode corresponds to a real eigenvalue leading to a real Ritz vector. The remaining modes represent the real and imaginary parts of complex Ritz vectors. The phase-shifted analogue of oscillatory mode 10 is mode 11 (not displayed). In figure 6, the corresponding amplitudes of the real modes are displayed.

Modes 1, 6 and 7 act as shift modes and resolve slow base-flow changes. This interpretation is corroborated by the behaviour of the mode amplitudes. The remaining modes describe vortex shedding $(i=2,3,4,5,10)$ or its second harmonics $(i=8,9)$. The oscillatory mode amplitudes are slowly growing, like the first vortex shedding pair (for $i=2,3$ ) and the second harmonics (for $i=8,9$ ), or slowly decaying (for $i=4,5,10)$. Intriguingly, the DMD modes describing vortex shedding have nearly identical frequencies and nearly identical shapes. This implies a redundancy which constitutes a challenge for reduced-order modelling.

By construction, the mode amplitudes have an exponentially growing or decaying envelope and can thus give no indication of initial or asymptotic values or temporal periods of maximum activity. In particular, an extrapolation beyond the sampling interval $t \in[30,120]$ is not meaningful. An additional potential challenge for reduced-order modelling is posed by the non-orthogonality of the DMD modes. 
(a)

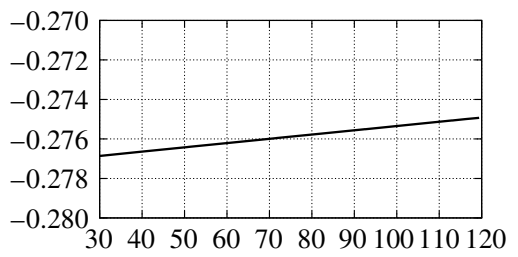

(c)

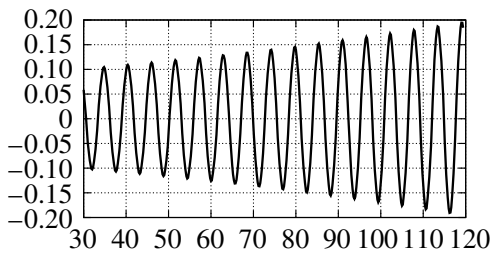

(e)

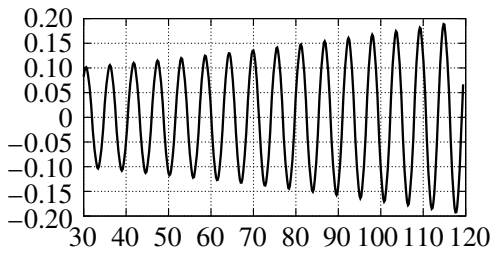

(g)



(i)

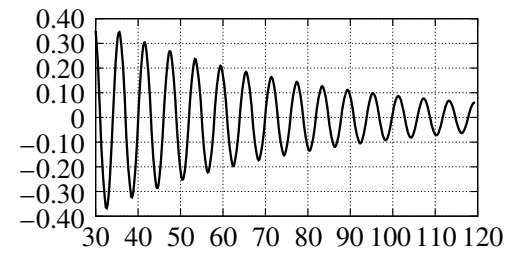

(b)

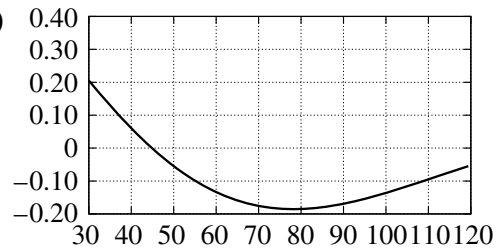

(d)

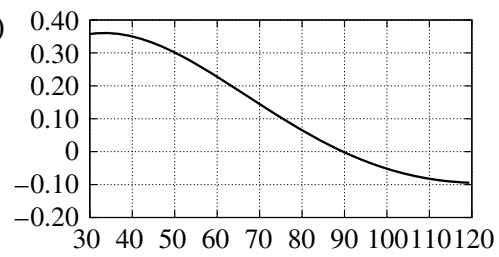

(f)

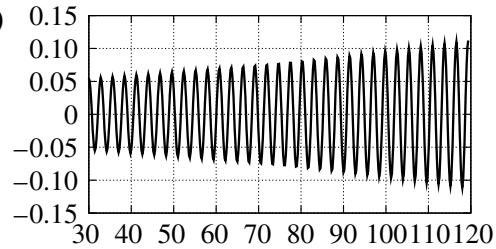

(h)

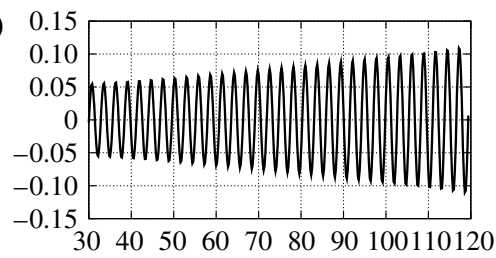

(j)

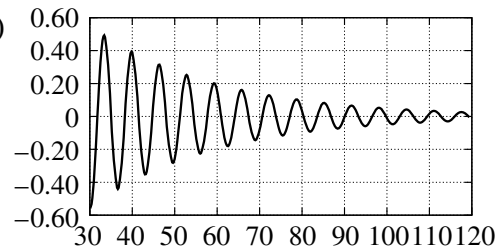

FIgURE 6. The same as figure 4 but for the first 10 DMD modes.

Already in the early literature (Rowley et al. 2009; Schmid 2010; Chen et al. 2012), DMD has been shown to accurately capture the onset of fluctuations in the linear regime or the post-transient behaviour on the attractor. The current results indicate difficulties of the DMD concerning the modal interpretation for a complete transient from the steady solution to the post-transient attractor. This issue has also been pointed out and analysed by Bagheri (2013). In the next section, we present an alternative DMD decomposition which addresses and removes the above-mentioned challenges.

\subsection{Recursive DMD}

In this section, the results of RDMD are presented. The procedure is demonstrated for the same set of snapshots as employed previously for POD and DMD. 
(a)

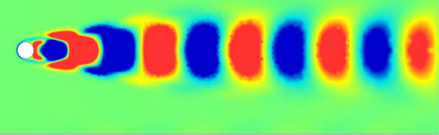

(c)

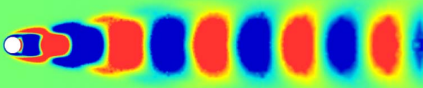

(e)

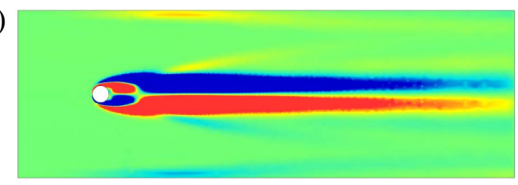

(g)

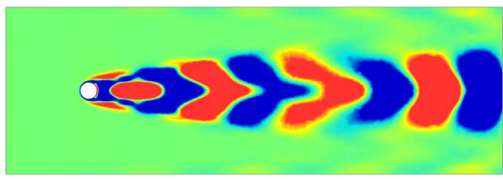

(i)

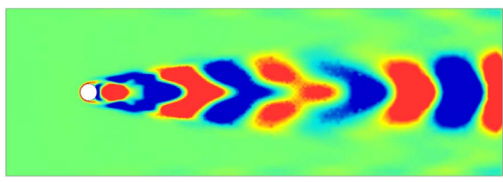

(b)

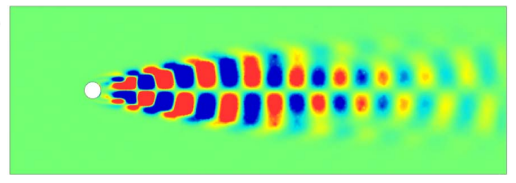

(d)

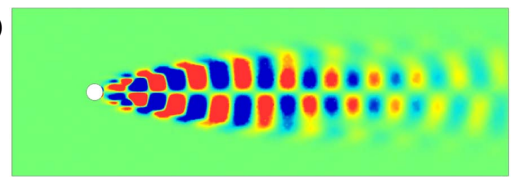

$(f)$

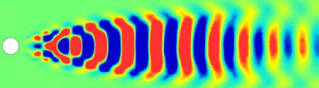

(h)

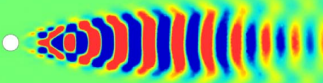

(j)

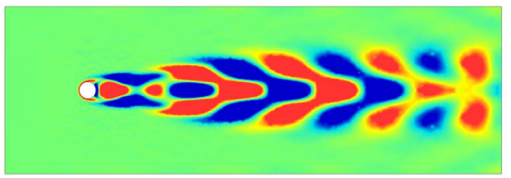

FIgURE 7. (Colour online) The same as figure 3 but for the first 10 RDMD modes.

The first 10 RDMD modes are depicted in figure 7. Intriguingly, RDMD resolves the first harmonics (modes $i=1,2)$, the second harmonics $(i=6,7)$ and the third harmonics $(i=8,9)$. The associated mode amplitudes (see figure 8) show corresponding oscillations starting near zero and reaching asymptotic values on the limit cycle. Mode 3 shows a nearly pure shift mode, describing the transition of the base flow from the steady solution to the time-averaged flow. In contrast to Noack et al. (2003), the amplitude becomes negative, since the sign of the RDMD mode is arbitrary. Modes 4 and 5 resolve intermediate vortex shedding patterns with maximum activity around $t=70$ and rather small residual fluctuations on the limit cycle. Modes 10 and 11 (not shown) are reminiscent of stability eigenmodes and peak near $t=55$, i.e. more than two periods earlier.

The first seven modes have significant similarities with the POD modes of figures 3 and 4. Yet, the oscillations are more symmetric (compare RDMD and POD mode 6) and show no apparent frequency mixing, as in POD modes $i=3,6,8$. Recursive DMD modes have by definition a lower averaged residual, compared with POD modes, but they look much cleaner and even reveal the third harmonics. It appears that RDMD modes have more in common with POD modes than with DMD modes. This should not come as a surprise, since the primary construction principle is an orthonormal decomposition leading to minimization of the residual, while the secondary criterion is the emulation of single-frequency DMD-mode behaviour. 
(a)



(c)

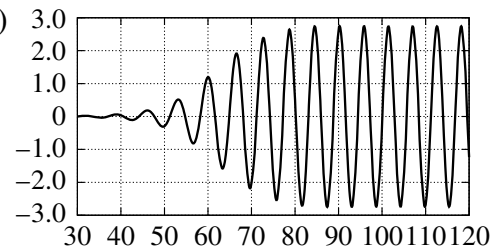

(e)

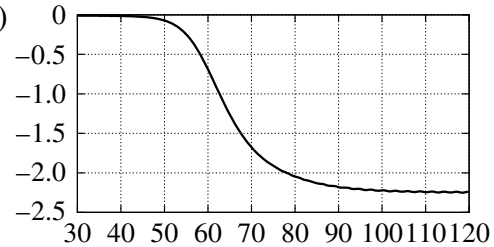

( $g$ )

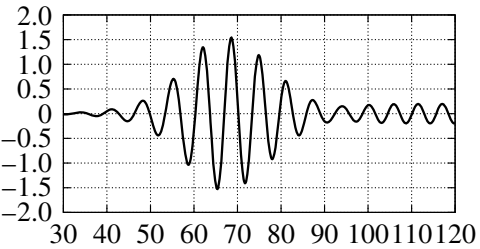

(i)



(b)

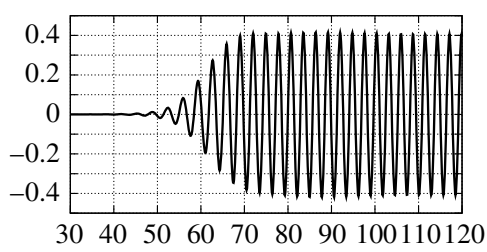

(d)

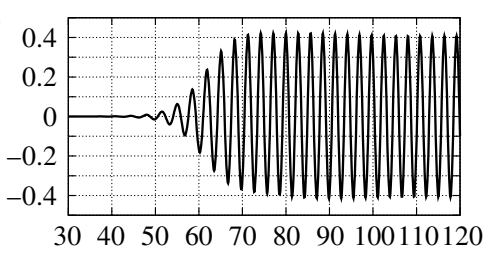

(f)

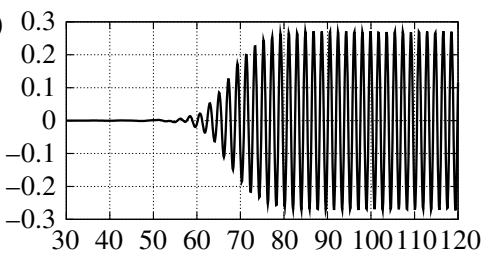

(h)



(j)

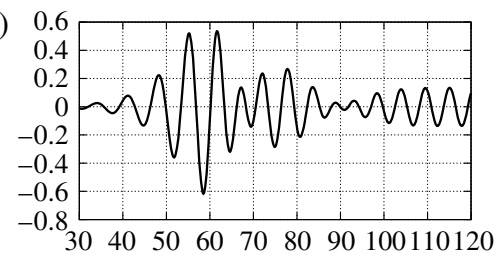

FIGURE 8 . The same as figure 4 but for the first 10 RDMD modes.

\subsection{Comparison of $P O D, D M D$ and $R D M D$}

In this section, results from POD, DMD and RDMD are quantitatively compared.

Figure 9 shows the truncation error of the POD, DMD and RDMD expansions as a function of the number of modes. As expected, all errors decrease monotonically with the number of modes, and POD outperforms DMD and RDMD. The RDMD residual, however, follows the POD value remarkably well and stays within similar orders of magnitude. In contrast, DMD approaches an effective asymptote near $10 \%$ of the final fluctuation level.

The temporal evolution of the instantaneous truncation error is displayed in figure 10 for POD, DMD and RDMD at $N=10$. The maximum value in $t \in[60,70]$ is lowest for POD and largest for DMD. Recursive DMD performs, as expected, 


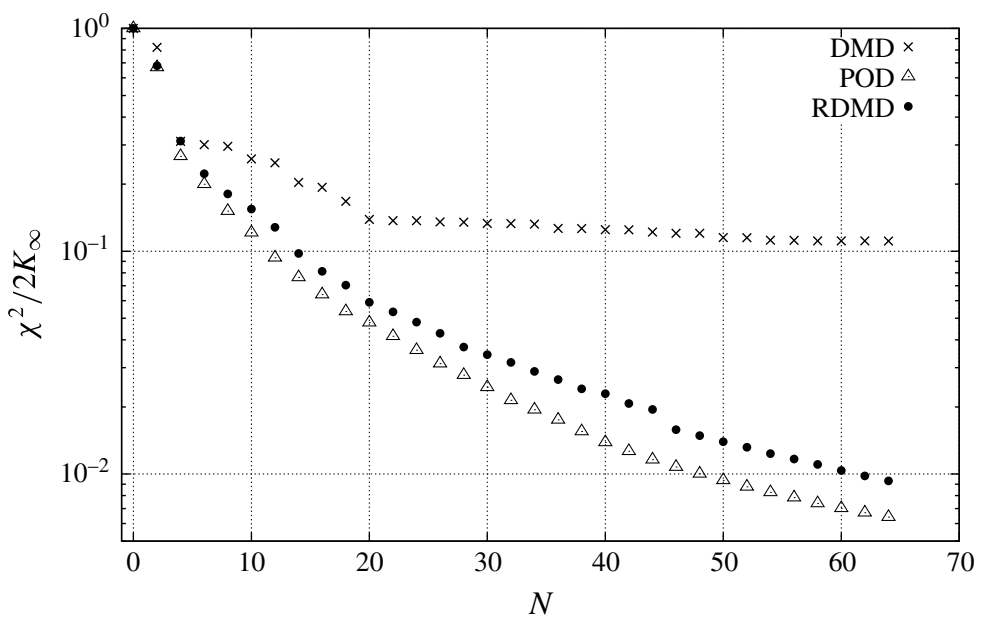

FIGURE 9. The time-averaged fluctuation level of the residual (3.11) for POD, DMD and RDMD with increasing number of modes. The value is normalized by the corresponding fluctuation level $\left(2 K_{\infty}\right)$ on the limit cycle.

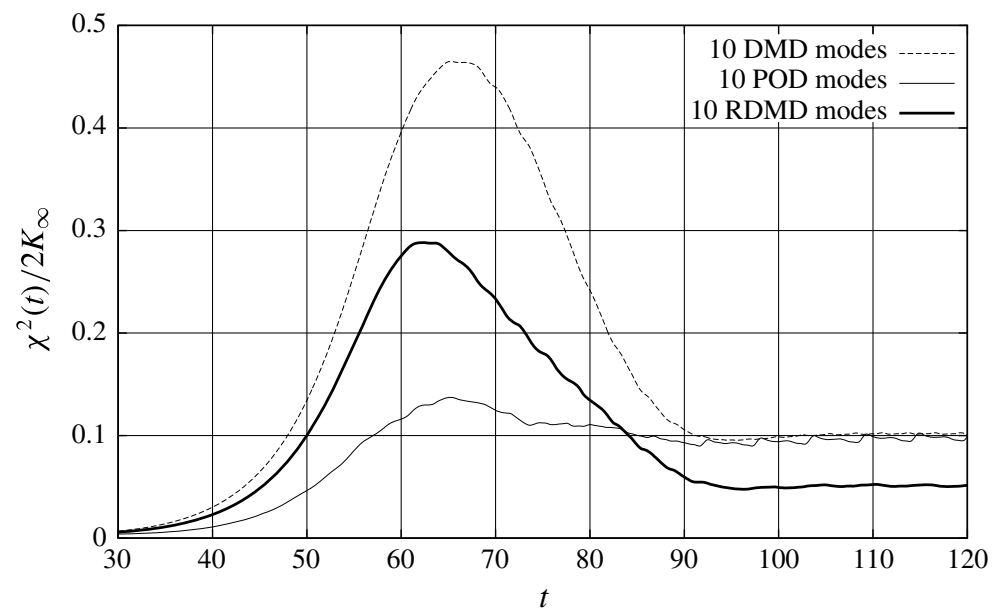

FIGURE 10. The instantaneous normalized truncation error (3.11) as a function of time for 10 POD, DMD and RDMD modes. The figure displays the sampling interval.

between the alternative expansions. A surprising feature is the asymptotes. Proper orthogonal decomposition and DMD have similar truncation errors near $10 \%$ of the terminal fluctuation level, while RDMD outperforms both with a final value at approximately $5 \%$. This is no contradiction to the optimal property of POD, as this property only guarantees a minimal time-averaged value, or, equivalently, a minimal value of the integral over the instantaneous truncation error, which it evidently has. The DMD-based frequency filtering included in RDMD appears to have 'anticipated' the limit-cycle behaviour. One indication of this 'anticipation' is the third harmonics which are featured in RDMD but absent in both POD and DMD. 
(a)

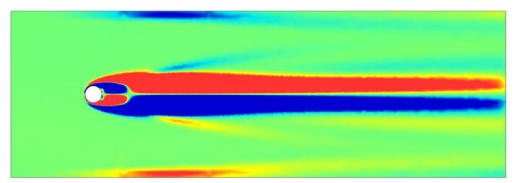

(c)

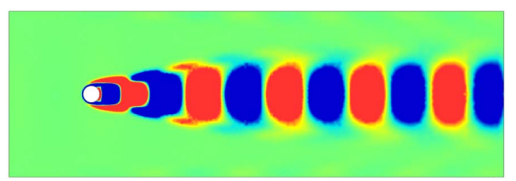

(e)

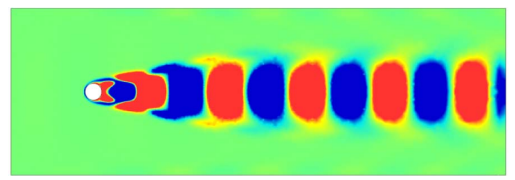

(g)

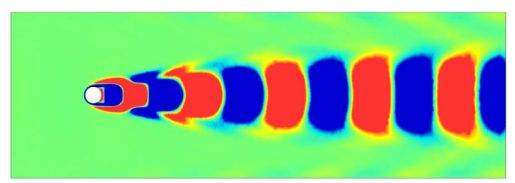

(i)

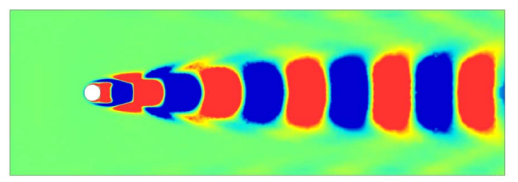

(b)

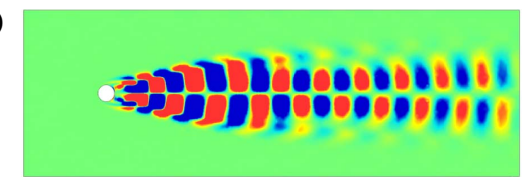

(d)



(f)

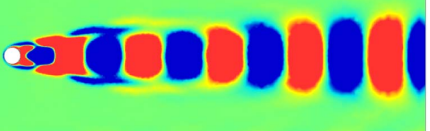

(h)

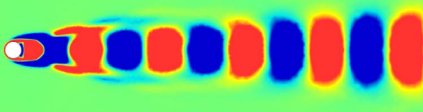

(j)

Figure 11. (Colour online) The same DMD modes as figure 5 but for the transient cylinder with $t \in[30 ; 120]$ and at $R e=120$.

At this stage, RDMD appears to be more suitable for reduced-order modelling when compared with POD or DMD. Like POD, RDMD yields orthonormal modes, but with purer frequency content. Like DMD, RDMD extracts oscillatory modes, but with well-defined initial and asymptotic behaviour of the mode amplitudes. In this sense, a strength of RDMD is that its amplitudes depart from oscillatory behaviour with exponential growth or decay of the DMD decomposition. In addition, the cycle-to-cycle variation of amplitude and frequency of RDMD coefficients is smaller than for similar POD mode coefficients.

The relative performance of POD, DMD and RDMD is rarely affected by a small change of the interval time under investigation. A numerical study with numerous time intervals indicates that the first modes keep their spatial structure but may have different energy content. Even significant changes of the interval only give rise to changes in the relative order of the modes. For instance, shifting the investigation interval to include more converged limit-cycle data decreases the energy content of slowly varying base-flow modes while higher harmonics become more dominant. In the extreme case of snapshots from the converged limit cycle, POD, DMD and RDMD become very similar. An opposite observation is made for intervals focusing on the initial transient. 
(a)

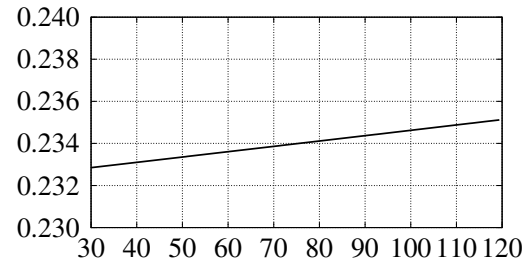

(c)

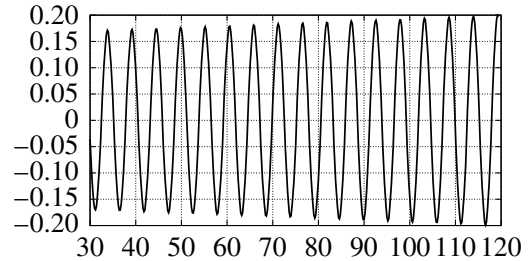

(e)

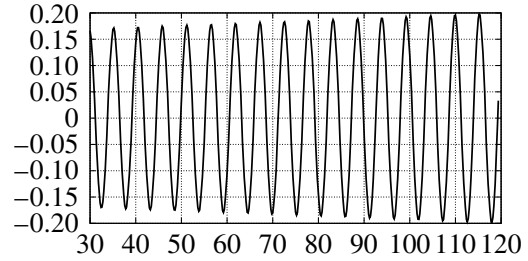

(g)

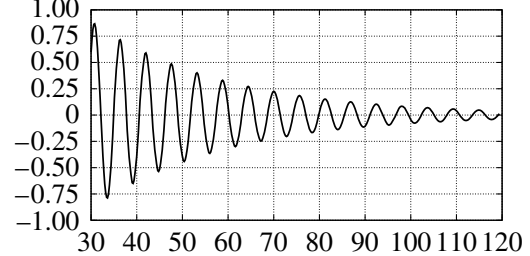

(i)



(b)

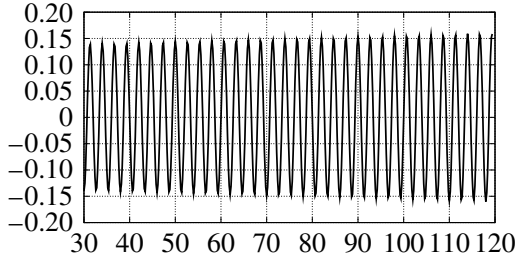

(d)

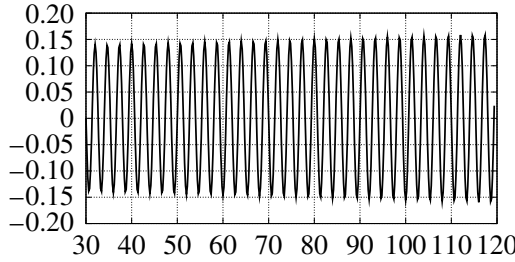

(f)

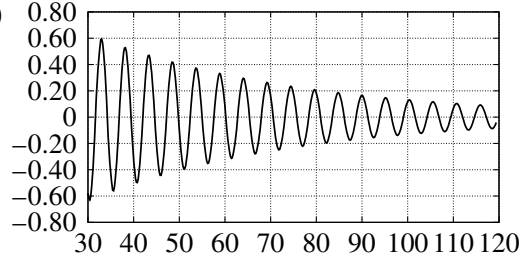

(h)

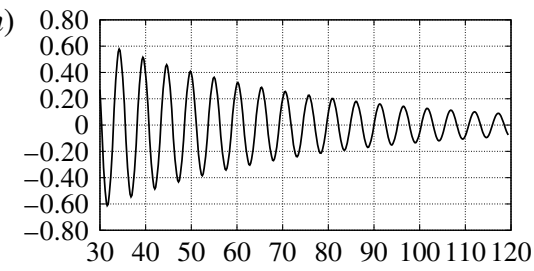

(j)

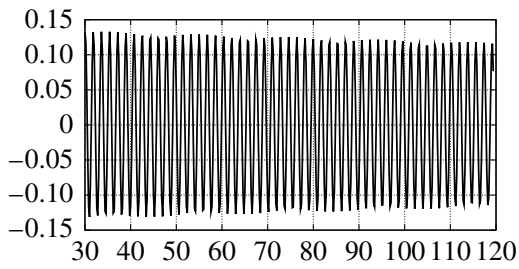

FIGURE 12. The modal amplitudes of the DMD modes of figure 11.

\subsection{Reconstruction of the flow at $R e=120$}

In the previous section (4.4), the data-driven Galerkin expansions have been evaluated for the training data. The residuals of these modal expansions are now tested for another data set: the transient cylinder wake at $R e=120$ with the same initial conditions during the time $t \in[30,120]$.

The change of the wake transient may be appreciated when the new DMD mode basis displayed in figure 11 is compared with the $R e=100$ reference in figure 5 . While the first five modes have similar frequency content (see figure 12), modes 6-10 are distinctly different. For instance, the quasi-steady modes 6 and 7 of the $R e=$ 100 reference have been replaced by the second harmonics for $R e=120$. The third harmonics do not appear in the top 10 of the reference data but are found as mode 10 for $R e=120$. 




FIGURE 13. The time-averaged fluctuation level of the residual (3.11) for POD, DMD and RDMD (obtained from the transient at $R e=100$ ), and DMD at $R e=120$ with increasing number of modes. The value is normalized by the corresponding fluctuation level $\left(2 K_{\infty}\right)$ on the limit cycle.

As cross-validation, the POD, DMD and RDMD expansions obtained from the transient wake at $R e=100$ are employed to resolve the transient wake at $R e=120$. First, the residuals for the whole transients are plotted as a function of the number of modes in figure 13. The residuals of POD and RDMD decrease similarly to a low fluctuation level as the number of modes increases to 64. The observation that RDMD outperforms POD particularly for high-order expansion is not a contradiction to the optimality property of POD, as the residual is determined on new data. Intriguingly, DMD shows a particularly poor performance compared with POD and RDMD. As expected, the residual of DMD decreases when the training data $(R e=100)$ are replaced by the testing data $(R e=120)$. However, we emphasize that RDMD based on training data at $R e=100$ performs much better for the new data at $R e=120$ compared with DMD obtained and tested for these new data. In other words, RDMD obtained for some operating condition appears to be robust against new data from changing operating conditions.

Figure 14 shows the residual for the four modal expansions of figure 13 as a function of time for a modal order of $N=10$. The maximum instantaneous residuals are ordered like the averaged residual values at $N=10$ : POD beats RDMD and RDMD beats DMD. The DMD obtained for the new transient data shows the largest maximum residual but is more accurate on the limit cycle.

Similar time-dependent residual behaviour is observed for expansions with more modes, except that RDMD is more often observed to outperform POD, keeping in mind that the expansions are obtained from $R e=100$ data but tested for the $R e=120$ transient.

\section{Modal decomposition of the wake past three rotating cylinders}

The performance of POD, DMD and RDMD is investigated for a non-periodic flow behind three rotating cylinders, which extends the previous cylinder wake simulations to a more complex configuration. All cylinders have unit diameter $D=1$. Their 




FIGURE 14. The instantaneous normalized truncation error (3.11) as a function of time for 10 POD, DMD and RDMD modes obtained for $R e=100$, and $10 \mathrm{DMD}$ modes from $R e=$ 120 transient data. The figure displays the same sampling interval as used for figure 13.

(a)

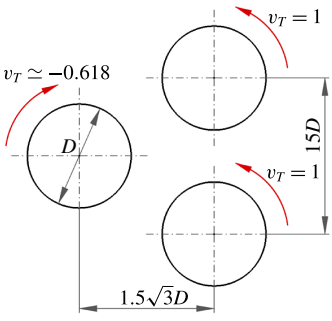

(b)



FIgURE 15. (Colour online) Flow past three rotating cylinders. Details of the test case (a) and the instantaneous vorticity field $(b)$.

centres are on the vertices of an equilateral triangle with an edge length of $1.5 D$. This triangle is centred at the origin and has one vertex on the negative $x$-axis and two vertices at same $x$-value downstream. The two rear cylinders rotate counterclockwise with tangential velocity $v_{T}=1$. The front cylinder rotates clockwise, and its tangential velocity $v_{T}=(\sqrt{5}-1) / 2 \approx 0.618$ (figure $\left.15 a\right)$. Due to the rotation speeds, the wake flow is intentionally not symmetrical. Figure $15(b)$ depicts the vorticity field.

In this section, we investigate the converged post-transient flow. The flow is computed with the same Navier-Stokes solver and the same accuracy as the cylinder wake in $\S 4$. The computational domain is bounded by the rectangle $-6 \leqslant x \leqslant 20$ $-y \leqslant 6 \leqslant y$ and is discretized by 4225 quadratic six-node elements. The Reynolds numbers, based on the diameter of a single cylinder, is 100 .

We are working at companion experiments for this configuration. The motivation is to have a simple geometry with three actuation inputs, the rotations of the cylinders, which can display complex dynamics but remains easily computable and experimentally realizable. The front cylinder controls the stagnation point while the rear cylinders manipulate each shear-layer individually. Thus, many drag-reducing mechanisms can be realized, like stagnation point stabilization (front cylinder), 
(a)

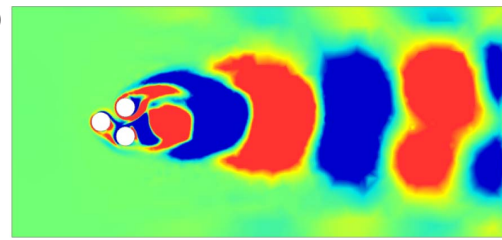

(c)

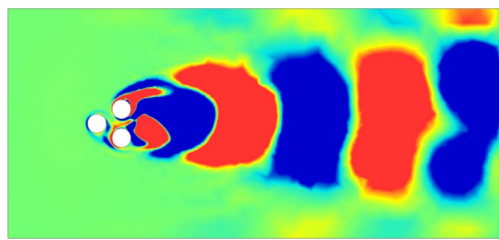

(e)

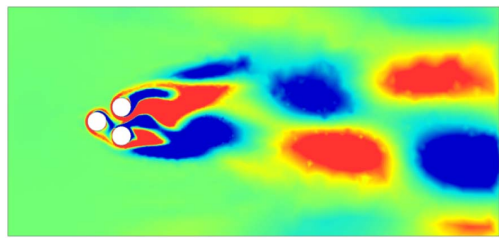

(g)

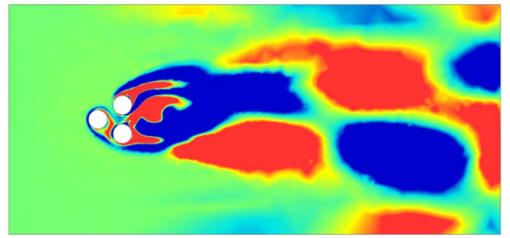

(i)

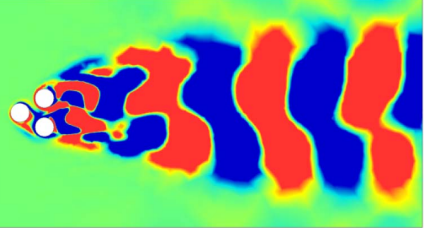

(b)

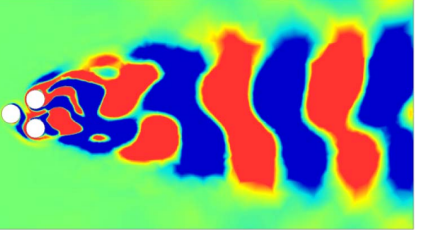

(d)

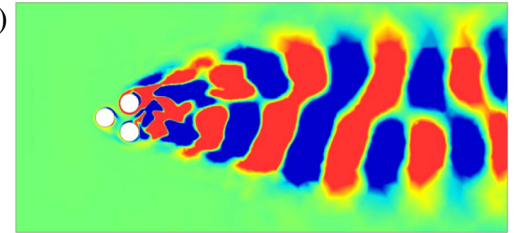

(f)

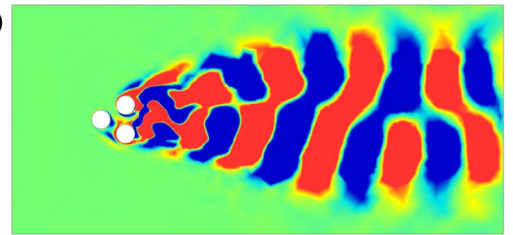

(h)

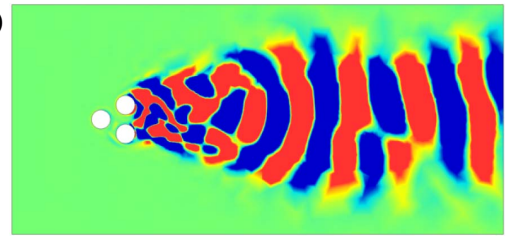

(j)

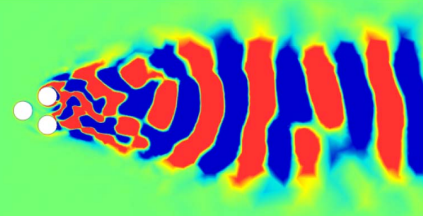

FIgURE 16. (Colour online) The first 10 POD modes of the flow past three rotating cylinders at $R e=100$. $(a, c, e, g, i)$ Modes $1-5,(b, d, f, h, j)$ modes 6-10. The vorticity of the mode is visualized in colour (green, zero; red, above a positive threshold; blue, below a negative threshold).

symmetric and antisymmetric high-frequency shear-layer excitation (rear cylinders) or base bleeding (rear cylinders generating a jet on the $x$-axis), to name a few.

Following the exposition of $\S 4$, we discuss POD ( $\$ 5.1)$, DMD ( $\$ 5.2)$ and RDMD ( $\$ 5.3)$, followed by a quantitative comparison of the residuals $(\S 5.4)$.

\subsection{Proper orthogonal decomposition}

The snapshots representing the attractor of the flow past three rotating cylinders are decomposed using POD. The first 10 of them, representing more than $99 \%$ of the fluctuation energy, are depicted in figure 16. The first two modes describe von Kármán vortex shedding and the next two modes resolve a low-frequency base-flow 
(a)

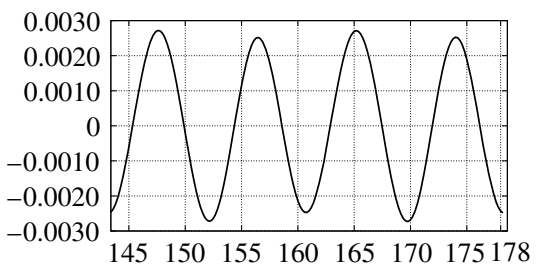

(c)

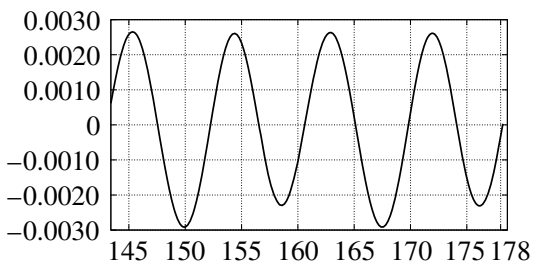

(e)



(g)

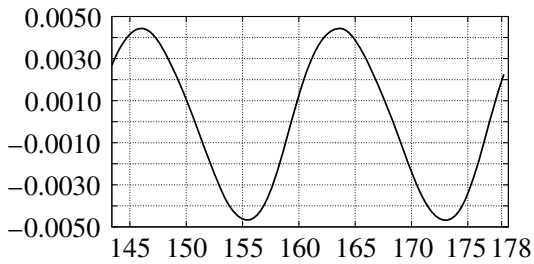

(i)

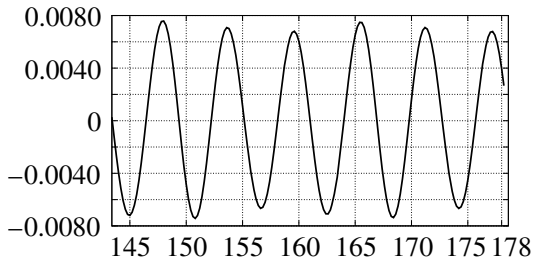

(b)

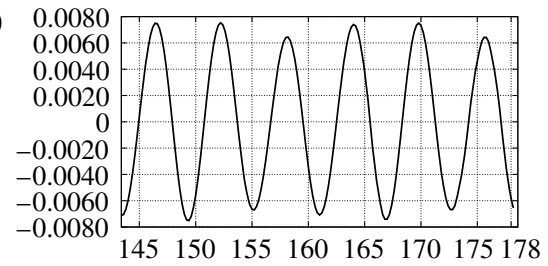

(d)

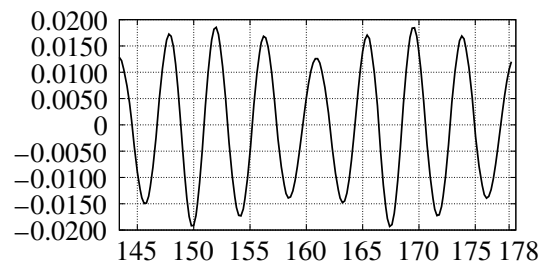

$(f)$

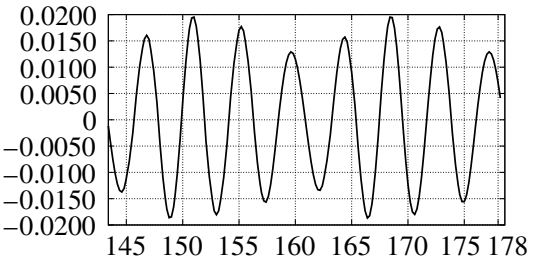

(h)

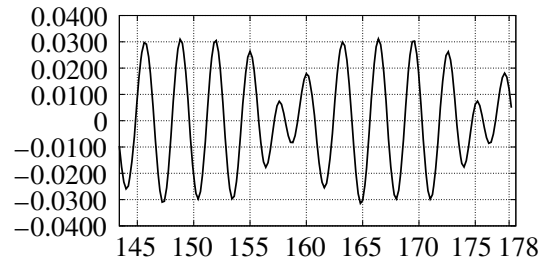

(j)

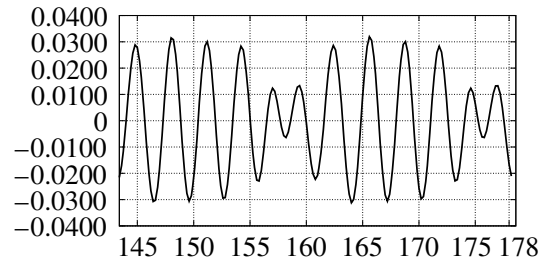

FIGURE 17. Flow past three rotating cylinders. The mode amplitudes of the POD modes of figure 16.

modulation. Modes 5-10 form pairs describing fluctuations at higher frequencies. The spatial mode interpretation is corroborated by the behaviour of the mode amplitudes in figure 17.

\subsection{Dynamic mode decomposition}

The same snapshots are subjected to DMD. The results, i.e. the eigenmodes and their amplitudes, are depicted in figures 18 and 19 respectively.

The modal amplitudes show, as expected, pure harmonic behaviour, while the nonsymmetric modes arise from a non-symmetrically forced wake. 
(a)

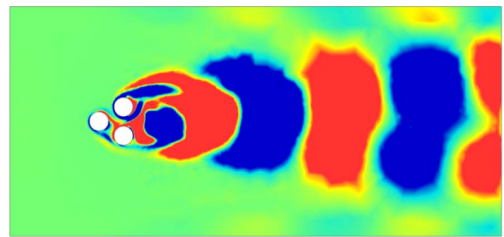

(c)

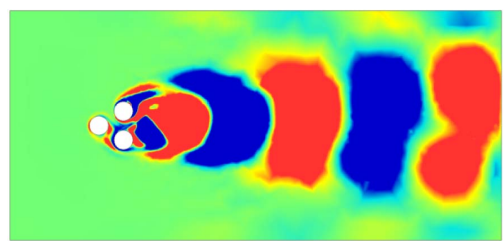

(e)

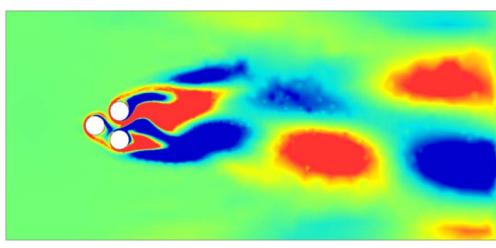

(g)

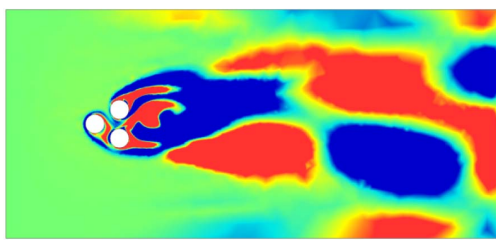

(i)

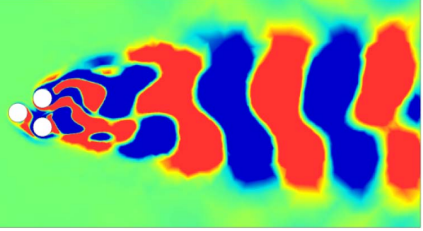

(b)

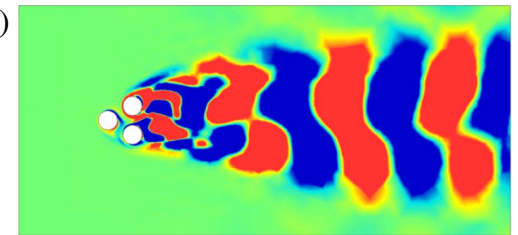

(d)

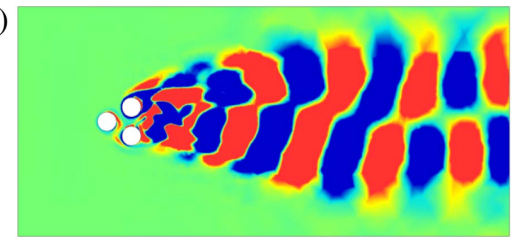

(f)

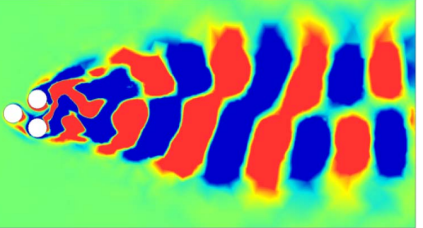

(h)

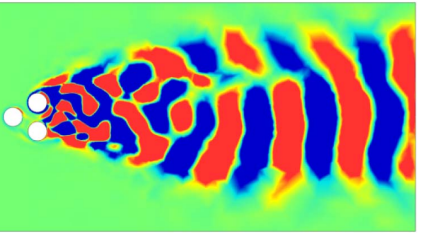

$(j)$

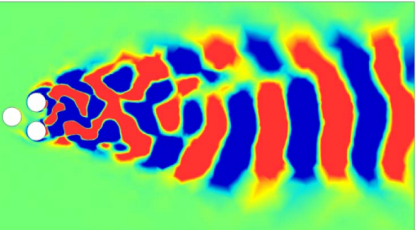

FIgURE 18. (Colour online) The same as figure 16 but for the first 10 DMD modes.

\subsection{Recursive DMD}

In this section, the results of RDMD are presented. Again, the procedure is demonstrated for the same set of snapshots as employed previously for POD and DMD. Figures 20 and 21 depict the modes and their amplitudes respectively. The frequency contents of POD and RDMD are very similar, while the amplitude modulation of POD is less pronounced in RDMD, corroborating the very purpose or RDMD, namely more harmonic modes.

\subsection{Comparison of $P O D, D M D$ and RDMD}

In this section, results from POD, DMD and RDMD are quantitatively compared. Figure 22 shows the time-averaged residual as a function of the number of modes 
(a)

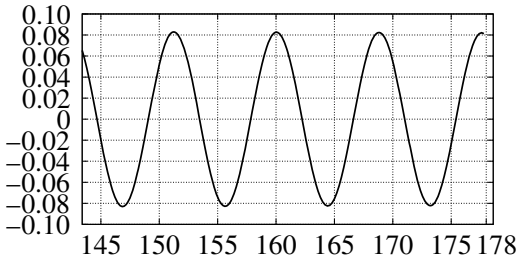

(c)

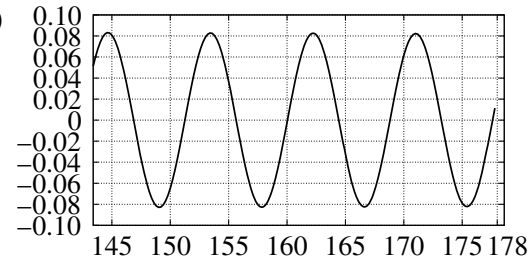

(e)

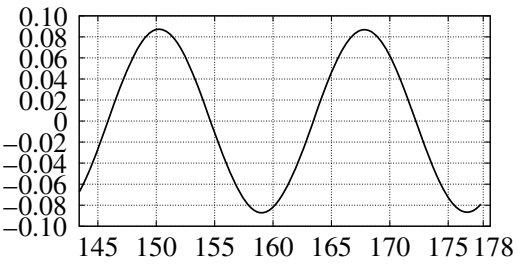

(g)

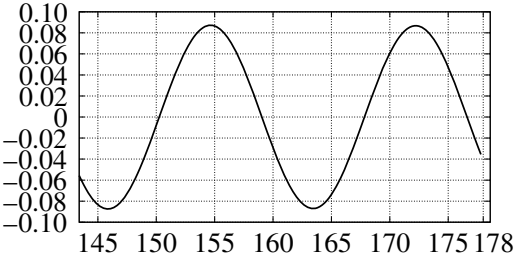

(i)

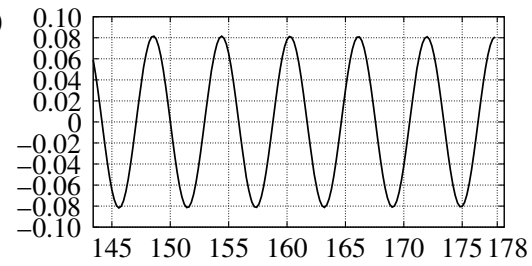

(b)

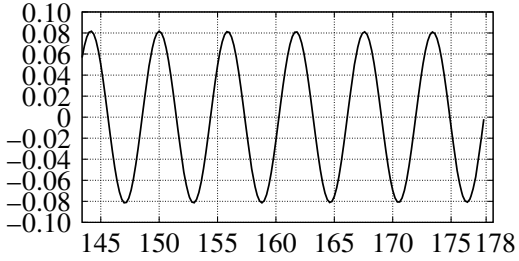

(d)

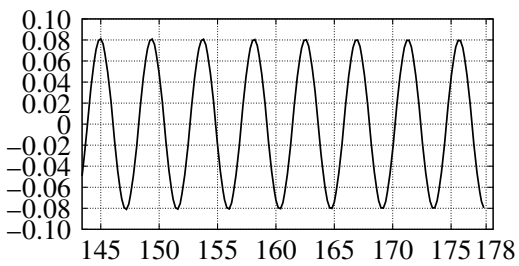

(f)

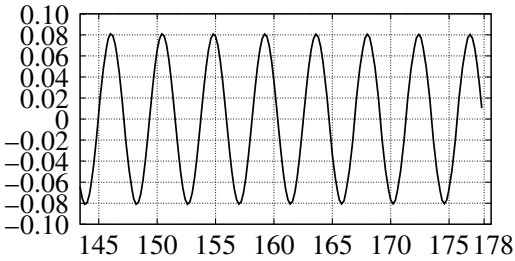

(h)

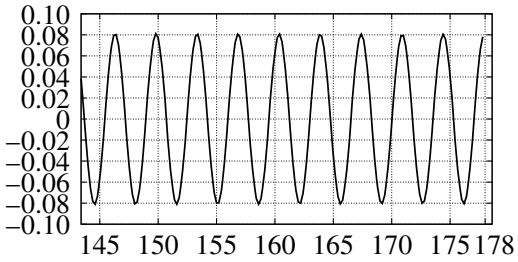

(j)

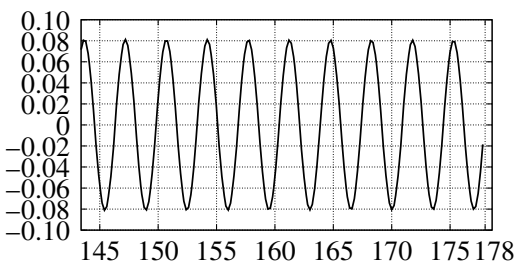

FIGURE 19. The same as figure 17 but for the first 10 DMD modes.

for all three expansions. The performance of RDMD follows POD closely, while the DMD residual is approximately one order of magnitude larger at $N=32$.

\section{Conclusions}

We propose a novel data-driven flow decomposition which combines the modal orthonormality and low truncation error $\chi^{2}$ (3.11) of POD with the frequency-distilling features of DMD. This decomposition is recursively defined. First, the data set is subjected to DMD, after which a normalized DMD mode is chosen which minimizes the averaged error $\chi^{2}$. This procedure is recursively repeated in the orthogonal subspace of computed modes. The resulting RDMD modes are orthonormal by 
(a)



(c)

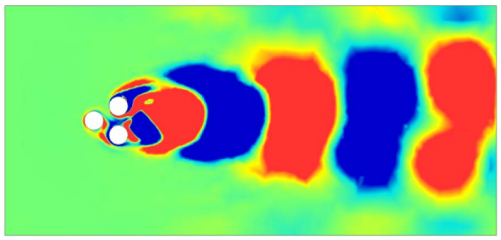

(e)



(g)

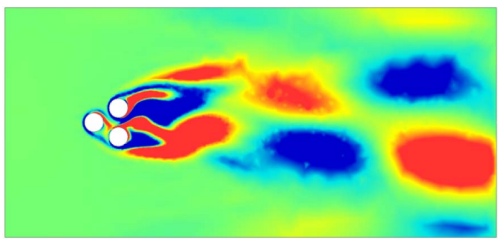

(i)

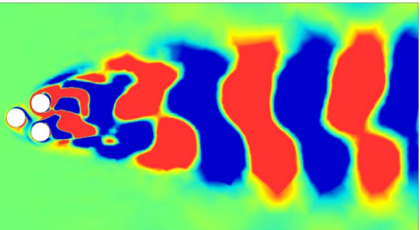

(b)

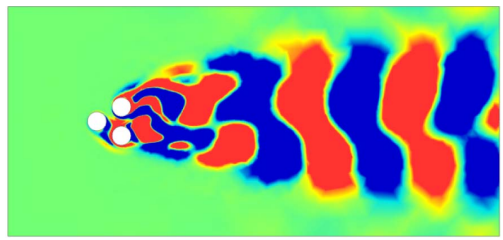

(d)

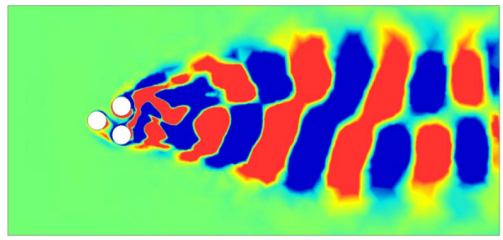

(f)



(h)

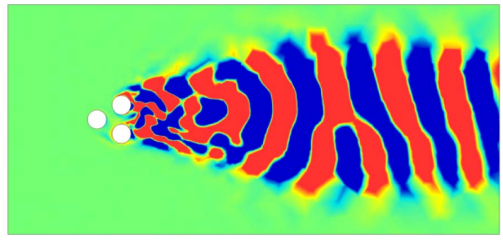

(j)

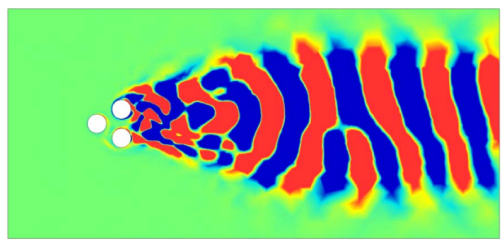

FIgURE 20. (Colour online) The same as figure 16 but for the first 10 RDMD modes.

construction and can be expected to have lower error $\chi^{2}$ than DMD modes while retaining the monochromatic features of DMD.

Proper orthogonal decomposition, DMD and RDMD have been applied to the same snapshots of a transient cylinder wake starting near the steady solution and terminating on the limit cycle. As expected, RDMD significantly outperforms DMD in terms of the maximum, time-averaged and asymptotic truncation error for all considered mode numbers by a large margin. In addition, the exponentially growing or decaying DMD amplitudes resemble neither initial nor asymptotic flow behaviour in a meaningful manner, while the RDMD amplitudes clearly identify the initial, transient and posttransient flow phases.

Also as expected, RDMD modes have far purer frequency content than POD modes but maintain the residual at a comparable level. While the maximum and average truncation errors of POD outperform RDMD, the latter shows a better resolution of 
(a)

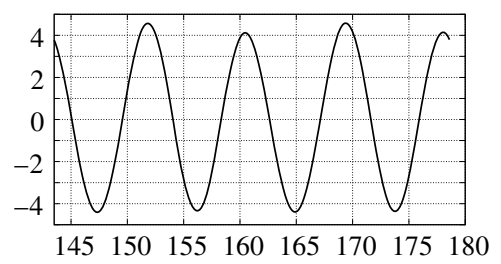

(c)

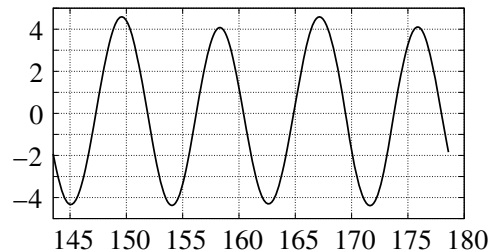

(e)

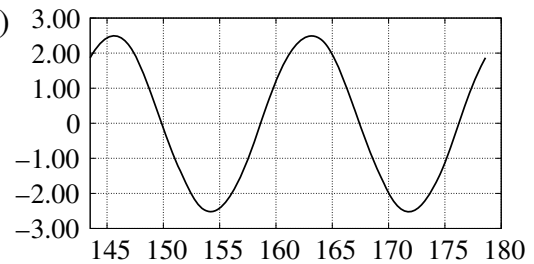

( $g$ )

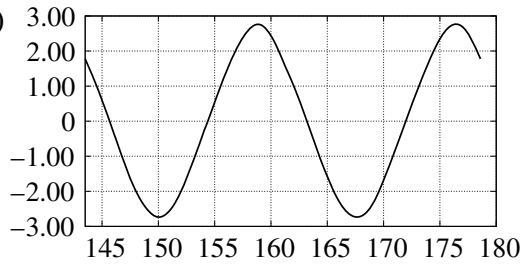

(i)

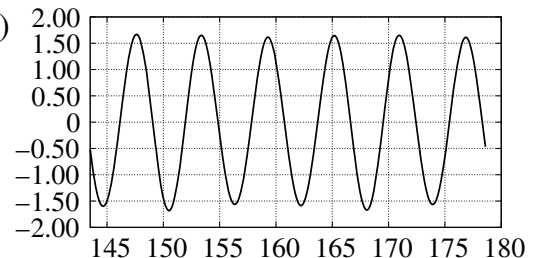

(b)

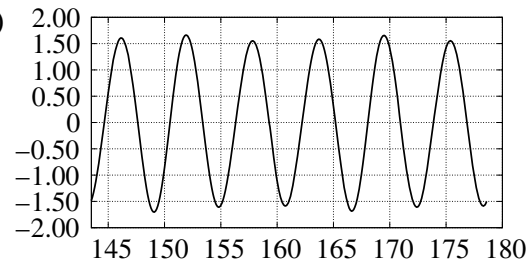

(d)

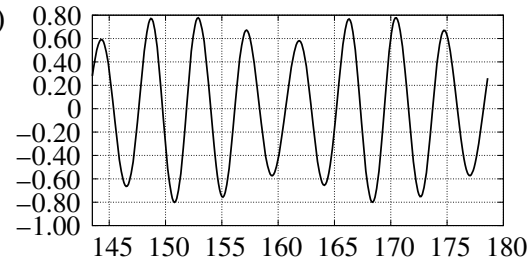

(f)

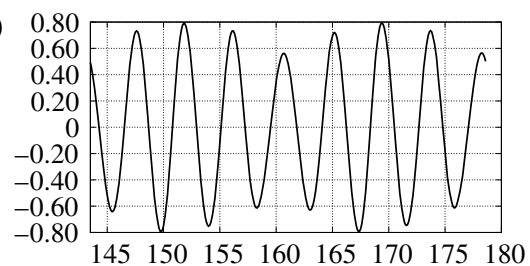

(h)

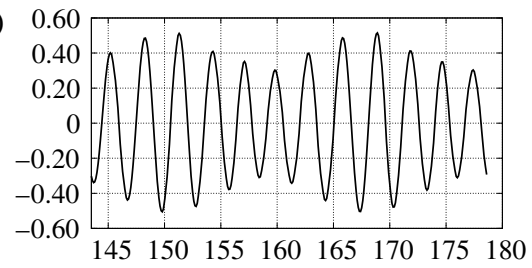

(j)

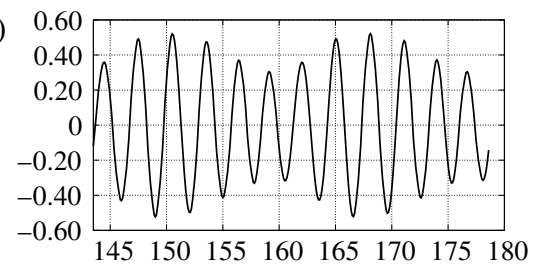

FIGURE 21. The same as figure 17 but for the first 10 RDMD modes.

the limit cycle: the asymptotic value for $N=10$ is half as large as the corresponding POD value, and the third harmonic frequency is only captured by RDMD in the first 10 modes. Table 1 provides a brief comparison of the main characteristics and features of POD, DMD and RDMD.

In the comparison, all modal decompositions were compared for the same data from which the modes were constructed. The robustness of the Galerkin expansions has also been tested for a transient at a higher Reynolds number of 120. Here, RDMD and POD performed very similarly, even better than DMD for the new data. A third investigation has been performed for a non-periodic and non-symmetric wake behind three rotating cylinders. Again, the main conclusions for RDMD, DMD and POD were corroborated. 




FIGURE 22. The time-averaged fluctuation level of the residual (3.11) for POD, DMD and RDMD for the flow past three rotating cylinders, with increasing number of modes. The value is normalized by the corresponding converged fluctuation level $\left(2 K_{\infty}\right)$ on the attractor.

Aspect

Ideal snapshots

Averaged truncation error

Maximum truncation error

Asymptotic truncation error

Modal frequency content

Noise sensitivity

Niche applications
POD

Uncorrelated

Optimal

$13 \%$

$10 \%$

Mixed

Low

Statistics
DMD

Time-resolved

Poor

$46 \%$

$10 \%$

Pure

High

without filter

Stability modes

Fourier modes

\section{RDMD}

Time-resolved

Good

$28 \%$

$5 \%$

Almost pure

Like

DMD

Transient

dynamics

TABLE 1. Comparison of POD, DMD and RDMD for the transient cylinder wake. The maximum and asymptotic truncation errors are for expansions with $N=10$ modes normalized with the post-transient fluctuation level $2 K_{\infty}$.

The literature contains alternative approaches for spectrally purified POD modes. One important recent contribution is spectral POD (Sieber, Paschereit \& Oberleithner 2016), which interpolates between POD and DMD by filtering the correlation matrix. This continuous interpolation offers an additional degree of freedom not present in RDMD; the price is the loss of strict orthonormality of the modes for all interpolation parameters. In a similar vein, Bourgeois, Martinuzzi \& Noack (2013) construct orthogonal POD modes with purer frequency content after Morlet-filtering the flow data at design frequencies. Recursive DMD can be considered as a simpler approach, which can be expected to perform well in an unsupervised manner, but leaves little room for tuning the modes for special purposes or applications. As a word of warning, all data-driven Galerkin expansions are - by definition - based on modes that are linear combinations of the snapshots, i.e. they cannot leave the space spanned by the training data. The benefit of one reduced-order representation method over another 
may actually be small. Noack (2016) sketches how future data-driven low-dimensional flow representations may be generalized to cure inherent limitations of the Galerkin expansion.

Summarizing, the current study indicates that RDMD is an attractive 'compromise' between POD and DMD. It sacrifices little of the optimal residual property of POD while retaining the single-frequency behaviour of DMD. Its potential in control-oriented reduced-order modelling will be explored in a future study. In addition, a particularly attractive opportunity for RDMD is the unsupervised extraction of generalized mean-field models with few dominant frequencies (Brunton \& Noack 2015).

\section{Acknowledgements}

B.N. acknowledges the funding and excellent working conditions of the Senior Chair of Excellence 'Closed-loop control of turbulent shear flows using reduced-order models' (TUCOROM 2010-2015) supported by the French Agence Nationale de la Recherche (ANR) and hosted by Institute PPRIME and the Collaborative Research Centre (CRC 880) 'Fundamentals of High Lift of Future Civil Aircraft' supported by the Deutsche Forschungsgemeinschaft (DFG) and hosted at the Technical University of Braunschweig. This work has also been funded by the Polish National Centre of Science under research grant no. DEC-2011/01/B/ST8/07264 'Novel Method of Physical Modal Basis Generation for Reduced Order Flow Models'. We have highly profited from stimulating discussions with M. Abel, S. Brunton, L. Cordier, R. Martinuzzi, B. Protas, R. Radespiel and R. Semaan. We thank the Ambrosys Ltd. Society for Complex Systems Management and the Bernd Noack Cybernetics Foundation for additional support.

\section{REFERENCES}

BAgheri, S. 2013 Koopman-mode decomposition of the cylinder wake. J. Fluid Mech. 726, 596-623. BARKLEY, D. \& Henderson, R. D. 1996 Three-dimensional Floquet stability analysis of the wake of a circular cylinder. J. Fluid Mech. 322, 215-241.

Bergmann, M. \& Cordier, L. 2008 Optimal control of the cylinder wake in the laminar regime by trust-region methods and POD reduced order models. J. Comput. Phys. 227, 7813-7840.

Bourgeois, J. A., Martinuzzi, R. J. \& NoAcK, B. R. 2013 Generalised phase average with applications to sensor-based flow estimation of the wall-mounted square cylinder wake. J. Fluid Mech. 736, 316-350.

BRUnton, S. L. \& NOACK, B. R. 2015 Closed-loop turbulence control: progress and challenges. Appl. Mech. Rev. 67 (5), 050801.

Chen, K. K., TU, J. H. \& Rowley, C. W. 2012 Variants of dynamic mode decomposition: boundary condition, Koopman, and Fourier analyses. J. Nonlinear Sci. 22 (6), 887-915.

Conts, C. M. 1997 Coherent structures in combustion. Prog. Energy Combust. Sci. 22, 427-509.

Courant, R. \& Hilbert, D. 1989 Methods of Mathematical Physics, vol. 1. Wiley-VCH.

Deane, A. E., Kevrekidis, I. G., Karniadakis, G. E. \& Orszag, S. A. 1991 Low-dimensional models for complex geometry flows: application to grooved channels and circular cylinders. Phys. Fluids A 3, 2337-2354.

Fletcher, C. A. J. 1984 Computational Galerkin Methods, 1st edn. Springer.

FöPPL, L. 1913 Wirbelbewegung hinter einen Kreiszylinder (transl.: vortex motion behind a circular cylinder). Sitzb. d. k. bayr. Akad. d. Wiss. 1, 1-18.

GALERKIN, B. G. 1915 Rods and plates: series occurring in various questions regarding the elastic equilibrium of rods and plates (translated). Vestn. Inzhen. 19, 897-908. 
Gerhard, J., Pastoor, M., King, R., Noack, B. R., Dillmann, A., Morzyński, M. \& TADMOR, G. 2003 Model-based control of vortex shedding using low-dimensional Galerkin models. In 33rd AIAA Fluids Conference and Exhibit, Orlando, Florida, USA, Paper 2003-4262.

HAN, Z.-H., StefAn, G. \& ZimmermanN, R. 2013 Improving variable-fidelity surrogate modeling via gradient-enhanced kriging and a generalized hybrid bridge function. Aerosp. Sci. Technol. 25 (1), 177-189.

Hoarau, C., Borée, J., Laumonier, J. \& Gervais, Y. 2006 Analysis of the wall pressure trace downstream of a separated region using extended proper orthogonal decomposition. Phys. Fluids 18, 055107.

Holmes, P., Lumley, J. L. \& Berkooz, G. 1998 Turbulence, Coherent Structures, Dynamical Systems and Symmetry, 1st edn. Cambridge University Press.

Jørgensen, B. H., SøRensen, J. N. \& BRøNS, M. 2003 Low-dimensional modeling of a driven cavity flow with two free parameters. Theor. Comput. Fluid Dyn. 16, 299-317.

VON KÁRMÁn, T. \& RUBACH, H. 1912 Über den Mechanismus des Flüssigkeits- und Luftwiderstandes. Phys. Zeitschr. XIII, 49-59.

Lorenz, E. N. 1956 Empirical orthogonal functions and statistical weather prediction. Tech. Rep.. MIT, Department of Meteorology, Statistical Forecasting Project.

Lorenz, E. N. 1963 Deterministic nonperiodic flow. J. Atmos. Sci. 20, 130-141.

Lugt, H. J. 1995 Vortex Flow in Nature and Technology. Krieger Publishing Company.

Lumley, J. L. 1967 The structure of inhomogeneous turbulent flows. In Atmospheric Turbulence and Wave Propagation (ed. A. M. Yaglom \& V. I. Tatarski), pp. 166-178.

MEZIĆ, I. 2013 Analysis of fluid flows via spectral properties of the Koopman operator. Annu. Rev. Fluid Mech. 45, 367-378.

NoACK, B. R. 2016 From snapshots to modal expansions - bridging low residuals and pure frequencies. J. Fluid Mech. 802, 1-4.

Noack, B. R., Afanasiev, K., Morzyński, M., Tadmor, G. \& Thiele, F. 2003 A hierarchy of low-dimensional models for the transient and post-transient cylinder wake. J. Fluid Mech. 497, 335-363.

Noack, B. R., Pelivan, I., Tadmor, G., Morzyński, M. \& Comte, P. 2004 Robust lowdimensional Galerkin models of natural and actuated flows. In Fourth Aeroacoustics Workshop, pp. 0001-0012. RWTH Aachen.

Oberleithner, K., Sieber, M., Nayeri, C. N., Paschereit, C. O., Petz, C., Hege, H.-C., NOACK, B. R. \& WYGNANSKI, I. 2011 Three-dimensional coherent structures in a swirling jet undergoing vortex breakdown: stability analysis and empirical mode construction. J. Fluid Mech. 679, 383-414.

ORSZAG, S. A. 1971 Numerical simulation of incompressible flows within simple boundaries: accuracy. J. Fluid Mech. 49, 75-112.

Rom-Kedar, V., Leonard, A. \& Wiggins, S. 1990 An analytical study of transport, mixing and chaos in unsteady vortical flow. J. Fluid Mech. 214, 347-394.

Rowley, C. W., Mezić, I., Bagheri, S., Schlatter, P. \& Henningson, D. S. 2009 Spectral analysis of nonlinear flows. J. Fluid Mech. 645, 115-127.

Schlegel, M., NoAck, B. R., Jordan, P., Dillmann, A., Gröschel, E., Schröder, W., Wei, M., Freund, J. B., Lehmann, O.\& TADmor, G. 2012 On least-order flow representations for aerodynamics and aeroacoustics. J. Fluid Mech. 697, 367-398.

SCHMID, P. J. 2010 Dynamic mode decomposition for numerical and experimental data. J. Fluid. Mech 656, 5-28.

Schumm, M., Berger, E. \& Monkewitz, P. A. 1994 Self-excited oscillations in the wake of two-dimensional bluff bodies and their control. J. Fluid Mech. 271, 17-53.

Sieber, M., PAschereit, C. O. \& Oberleithner, K. 2016 Spectral proper orthogonal decomposition. J. Fluid Mech. 792, 798-828.

Siegel, S. G., Seidel, J., Fagley, C., Luchtenburg, D. M., Cohen, K. \& Mclaughlin, T. 2008 Low dimensional modelling of a transient cylinder wake using double proper orthogonal decomposition. J. Fluid Mech. 610, 1-42. 
Sirovich, L. 1987 Turbulence and the dynamics of coherent structures. Part I: coherent structures. Q. Appl. Maths XLV, 561-571.

SuH, Y. K. 1993 Periodic motion of a point vortex in a corner subject to a potential flow. J. Phys. Soc. Japan 62, 3441-3445.

TAYLOR, C. \& HoOd, P 1973 A numerical solution of the Navier-Stokes equations using the finite element technique. Comput. Fluids 1 (1), 73-100.

Williamson, C. H. K. 1996 Vortex dynamics in the cylinder wake. Annu. Rev. Fluid Mech. 28, 477-539.

ZeBIB, A. 1987 Stability of viscous flow past a circular cylinder. J. Engng Maths 21, 155-165.

Zhang, H.-Q., Fey, U., Noack, B. R., König, M. \& Eckelmann, H. 1995 On the transition of the cylinder wake. Phys. Fluids 7 (4), 779-795. 